\title{
Sum rules and spectral patterns of dichroism in inner-shell photoelectron spectra
}

\author{
A. Verweyen, ${ }^{1, *}$ A. N. Grum-Grzhimailo, ${ }^{2}$ and N. M. Kabachnik ${ }^{2}$ \\ ${ }^{1}$ II. Institut für Experimentalphysik, Universität Hamburg, Luruper Chaussee 149, D-22761 Hamburg, Germany \\ ${ }^{2}$ Institute of Nuclear Physics, Moscow State University, Moscow 119899, Russia
}

(Received 31 December 1998)

\begin{abstract}
A theoretical description of dichroism in the photoelectron spectra of atoms polarized in different directions is presented. It is based on the general expression for the photoelectron angular distribution written in $L S J$ approximation. We show that, for photon energies far above threshold, the spectral distribution of dichroism in the photoelectron spectrum in most cases does not depend on the photoionization dynamics; for atoms with $S$ symmetry, simple spectral patterns are obtained. Useful sum rules are given for the dichroism integrated over groups of photoelectron multiplet lines. Recent experimental data on magnetic dichroism and linear dichroism in the nonresonant inner-shell spectra of $\mathrm{Cr}$ ( $3 p$ shell), $\mathrm{Eu}(4 f$ shell), and $\mathrm{Fe}(3 p$ shell) are analyzed. An outlook is given on the dichroism in the region of autoionization resonances. [S1050-2947(99)10808-4]
\end{abstract}

PACS number(s): $32.80 . \mathrm{Fb}, 32.80 . \mathrm{Hd}$

\section{INTRODUCTION}

Circular and linear dichroism in the photoelectron angular distribution of free polarized atoms yield detailed information on the structure of atoms and their interaction with the electromagnetic field. In principle, dichroism measurements can lead to the realization of a so-called "complete" experiment, i.e., the determination of all photoionization amplitudes $[1,2]$. The pioneering experiment in the vacuum ultraviolet (vuv) has been performed on laser-polarized $\mathrm{Yb}$ with an ArI lamp [3]. Progress on the way to a complete experiment has recently been reported for atomic photoionization with synchrotron radiation [4-6]. Dichroism in inner-shell photoelectron spectra is a very promising tool for the element-specific determination of magnetic properties from thin films and multilayers. Since the inner-shell photoionization in solids is strongly influenced by local interactions, atomic models can be successfully used in many cases as a first step for a qualitative understanding of the dichroism spectra of atoms bound to a surface ([7-12], and references therein).

The vuv photoelectron spectra of open-shell atoms generally display a pronounced multiplet structure which is well described by $L S J$ coupled final ionic states (in the $L S J$ coupling approximation, the state of an atom is described by the quantum numbers $L S J$, where the total angular momentum $L$ is coupled with the total spin $S$ to the total angular momentum $J$ ). The $L S J$ approximation is very well fulfilled for the $3 p$ shell of the $3 d$ transition metal atoms $[13,14]$ and the $4 d$ shell of the rare earth atoms [8] because of the very strong exchange interaction of the inner vacancy and the open $3 d / 4 f$ shell, respectively. The spin-orbit splitting, which is much smaller than the exchange splitting, leads to multiplet fine structure. Similarly, $L S J$ coupling is a reasonable first approximation for the description of the $3 s$ and $3 d$ spectra of the $3 d$ transition metal atoms and the $4 f, 5 s, 5 p$ spectra of the rare earth atoms; however, deviations become significant

*Present address: Hamburger Synchrotronstrahlungs-Labor HASYLAB at DESY, Notkestr. 85, D-22603 Hamburg, Germany. for the heavy lanthanides. In order to provide a firm basis for the analysis of experimental spectra, we extend an earlier work [15] on the angular distribution of photoelectrons from polarized atoms by introducing the nonrelativistic $L S J$ coupling approximation. Further simplification is achieved by using the single-configuration approximation. Special emphasis is placed on general patterns and sum rules.

Dichroism in the photoelectron spectra of polarized atoms is the intensity difference for two different orientations either of the photon polarization or of the target atom polarization. Conventionally dichroism is related to changes in the light polarization for one and the same state of the target polarization ([16], and references therein). Following the experiment of Baumgarten et al. [17], dichroism due to the difference in the target polarization (for one and the same light polarization) has also been investigated. In this paper we consider only dichroism due to changes of the target atom polarization.

In the following we discuss a number of commonly performed dichroism measurements, namely circular magnetic dichroism (CMD), linear magnetic dichroism in the angular distribution (LMDAD), and linear dichroism (LD). CMD and LMDAD are measured with circularly and linearly polarized ionizing radiation, respectively, for two mutually antiparallel directions of the atomic polarization. Only the orientation of the target (but not the alignment) contributes to the magnetic dichroism [10]. On the other hand, LD is measured with linearly polarized light for mutually perpendicular directions of the atomic polarization. ${ }^{1}$

In general, both the alignment and the orientation of the target atom contribute to LD. Usually, however, the contribution of atomic orientation is negligible due to either the experimental geometry [10] or the method used to polarize the target atoms; for example, atoms prepared by optical pumping with linearly polarized laser radiation are aligned

\footnotetext{
${ }^{1}$ It should not be confused with dichroism due to a change in the linear polarization of the ionizing radiation, which is also abbreviated as LD in the literature [16].
} 


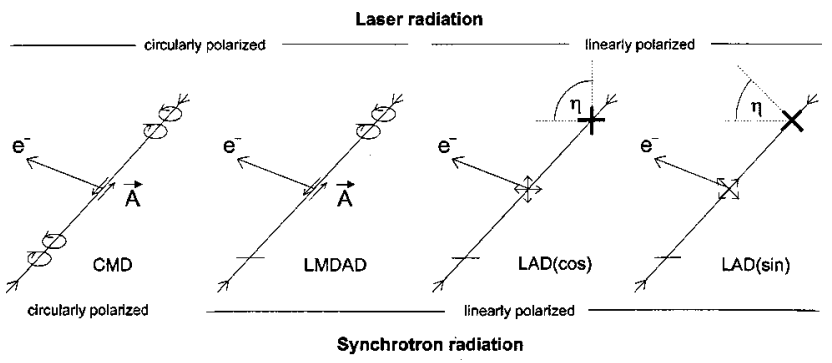

FIG. 1. Possible experimental geometries for the measurement of different types of dichroism. The vector $\vec{A}$ of the atomic orientation (left panels) is pointing into the direction of the photon spin.

but not oriented. It is assumed here that the experimental conditions are chosen in a way that only alignment contributes to LD. To avoid any confusion we refer to this kind of dichroism as linear alignment dichroism (LAD). Note that we use the terms CMD and LAD both for angle-resolved and angle-integrated experiments.

For illustration, Fig. 1 displays possible geometries for the measurement of CMD, LMDAD, and LAD with synchrotron radiation, where the polarized atoms are prepared by laser pumping. The vector $\vec{A}$ of the atomic orientation (left panels) is pointing in the direction of the photon spin for circularly polarized laser radiation. For linearly polarized laser radiation (right panels) the axis $\vec{A}$ of the atomic alignment is directed along the electric-field vector of the laser radiation. The angle $\eta$ between the polarization vectors of the laser and the synchrotron radiation can be varied by means of an optical polarizer. The variation of $\eta$ allows the measurement of a number of independent LAD quantities. The two leading quantities $\mathrm{LAD}(\cos )$ and $\mathrm{LAD}(\sin )$, where the notation indicates a $\cos / \mathrm{sin}$ dependence on the angle $2 \eta[18]$ can, for example, be determined as indicated in Fig. 1 (right panels). It should be noted that the conclusions obtained in this paper are, in most cases, independent of the specific experimental geometry.

The paper is organized in the following way. First we incorporate the nonrelativistic approximation (Sec. II A) and, in a second step, the single-configuration approximation (Sec. II B) into the general theory of the angular distribution of photoelectrons from polarized atoms. The results obtained in this way imply very general properties of the dichroism in nonresonant photoelectron spectra, which are presented in Sec. III. In Sec. IV a number of recently published nonresonant dichroism experiments on free metal atoms are analyzed with respect to our theoretical results; an experiment in the region of autoionization resonances is also briefly discussed.

Atomic units are used throughout this paper unless otherwise indicated.

\section{ANGULAR DISTRIBUTION OF PHOTOELECTRONS FROM POLARIZED ATOMS IN THE NONRELATIVISTIC APPROXIMATION}

\section{A. General treatment}

We consider the photoionization process in the dipole approximation where the atom in the initial state is characterized by a well-defined total angular momentum $J_{0}$ and other quantum numbers $\alpha_{0}$. The emitted photoelectron has a ki- netic energy $\varepsilon$ and orbital and total angular momenta $l$ and $j$, respectively. The final ionic state is characterized by the total angular momentum $J_{f}$ and other quantum numbers $\alpha_{f}$. The process can thus be written as

$$
\alpha_{0} J_{0}+\gamma \rightarrow\left[\alpha_{f} J_{f}+\varepsilon l j\right] J .
$$

In Eq. (1) $J$ denotes the total angular momentum of the final system (ion+electron). The possible values of $J$ are determined by the dipole selection rules.

The cross section of this photoionization process is given by the general theory of the angular distribution of photoelectrons from polarized atoms for arbitrarily polarized light $[1,2,15,19]$ and can be presented in the form [15]

$$
\frac{d \sigma}{d \Omega}=\pi \alpha \omega\left(3 \hat{J}_{0}\right)^{-1} \sum_{k_{0} k k_{\gamma}} \rho_{k_{0} 0} B_{k_{0} k k_{\gamma}} F_{k_{0} k k_{\gamma}},
$$

where $\omega$ is the frequency of the ionizing photon and $\alpha$ denotes the fine-structure constant; the notation $\hat{J}_{0} \equiv\left(2 J_{0}\right.$ $+1)^{1 / 2}$ has been introduced for brevity. The statistical tensors (state multipoles) $\rho_{k_{0} 0}$ with rank $k_{0}=0,1, \ldots, 2 J_{0}$ describe the atomic polarization of the initial state in the coordinate system with $z$ axis along the polarization axis of the target atom. The geometrical factors

$$
\begin{aligned}
F_{k_{0} k k_{\gamma}}= & \sum_{q_{\gamma}} 4 \pi\left\{Y_{k_{0}}\left(\theta_{a}, \phi_{a}\right) \otimes Y_{k}\left(\theta_{e}, \phi_{e}\right)\right\}_{k_{\gamma} q_{\gamma}} \\
& \times \rho_{k_{\gamma} q_{\gamma}}\left(p_{1}, p_{2}, p_{3}\right)
\end{aligned}
$$

contain the direction of the atomic polarization $\left(\theta_{a}, \phi_{a}\right)$ and the direction of the photoelectron emission $\left(\theta_{e}, \phi_{e}\right)$ in the laboratory frame, and the polarization state of the photon which is characterized by the statistical tensors $\rho_{k_{\gamma} q_{\gamma}}$ which, in turn, depend on the Stokes parameters $p_{1}, p_{2}, p_{3}$. The tensorial product of spherical harmonics in Eq. (3) is given by

$$
\begin{aligned}
& \left\{Y_{k_{0}}\left(\theta_{a}, \phi_{a}\right) \otimes Y_{k}\left(\theta_{e}, \phi_{e}\right)\right\}_{k_{\gamma} q_{\gamma}} \\
& \quad=\sum_{q_{0} q}\left(k_{0} q_{0}, k q \mid k_{\gamma} q_{\gamma}\right) Y_{k_{0} q_{0}}\left(\theta_{a}, \phi_{a}\right) Y_{k q}\left(\theta_{e}, \phi_{e}\right),
\end{aligned}
$$

where $\left(k_{0} q_{0}, k q \mid k_{\gamma} q_{\gamma}\right)$ is a Clebsch-Gordan coefficient. The coefficients $B_{k_{0} k k_{\gamma}}$, which contain the dipole amplitudes, describe the dynamics of the photoionization process; they are given by [15]

$$
\begin{aligned}
B_{k_{0} k k_{\gamma}}= & 3 \hat{J}_{0} \sum_{l l^{\prime} j j^{\prime} J J^{\prime}}(-1)^{J+J_{f}+k_{\gamma}-1 / 2} \hat{J} \hat{J}^{\prime} \hat{j} \hat{j}^{\prime} \hat{l} \hat{l}^{\prime}\left(l 0 l^{\prime} 0 \mid k 0\right) \\
& \times\left\{\begin{array}{ccc}
j & l & \frac{1}{2} \\
l^{\prime} & j^{\prime} & k
\end{array}\right\}\left\{\begin{array}{ccc}
j & J & J_{f} \\
J^{\prime} & j^{\prime} & k
\end{array}\right\}\left\{\begin{array}{ccc}
J_{0} & 1 & J \\
J_{0} & 1 & J^{\prime} \\
k_{0} & k_{\gamma} & k
\end{array}\right\} \\
& \times\left\langle\alpha_{f} J_{f}, l j: J\|D\| \alpha_{0} J_{0}\right\rangle\left\langle\alpha_{f} J_{f}, l^{\prime} j^{\prime}: J^{\prime}\|D\| \alpha_{0} J_{0}\right\rangle^{*} .
\end{aligned}
$$

The standard notation for the Wigner $6 j$ and $9 j$ coefficients is used. The multichannel dipole amplitudes 
$\left\langle\alpha_{f} J_{f}, l_{j}: J\|D\| \alpha_{0} J_{0}\right\rangle$ contain the incoming-wave-normalized wave function, corresponding to an asymptotic channel with quantum numbers $\alpha_{f}, J_{f}, l, j, J$.

The differential cross section Eq. (2) can be written in an equivalent but more conventional form [15]:

$$
\frac{d \sigma}{d \Omega}=\frac{\sigma}{4 \pi}\left[1+\sum_{k_{0} k k_{\gamma}} \mathcal{A}_{k_{0} 0} \beta_{k_{0} k k_{\gamma}} F_{k_{0} k k_{\gamma}}\right],
$$

where $\sigma$ is the angle-integrated photoionization cross section of an unpolarized atom:

$$
\sigma=\frac{4 \pi^{2} \alpha \omega}{3\left(2 J_{0}+1\right)} \sum_{l j J}\left|\left\langle\alpha_{f} J_{f}, l j: J\|D\| \alpha_{0} J_{0}\right\rangle\right|^{2} .
$$

$\mathcal{A}_{k_{0} 0}=\rho_{k_{0} 0} / \rho_{00}$ are the reduced statistical tensors of the initial state. The summation in Eq. (6) is performed over all possible sets $\left(k_{0} k k_{\gamma}\right)$ except $(000)$. The generalized anisotropy coefficients $\beta_{k_{0} k k_{\gamma}}$ are related to the coefficients $B_{k_{0} k k_{\gamma}}$ of Eq. (5) by

$$
\beta_{k_{0} k k_{\gamma}}=\sqrt{3} \frac{B_{k_{0} k k_{\gamma}}}{B_{000}} .
$$

The monopole coefficient $B_{000}$ is given by

$$
B_{000}=\sqrt{3} \sum_{l j J}\left|\left\langle\alpha_{f} J_{f}, l j: J\|D\| \alpha_{0} J_{0}\right\rangle\right|^{2}
$$

From Eqs. (7)-(9) it is clear that the coefficients $\beta_{k_{0} k k_{\gamma}}$ correspond to the coefficients $B_{k_{0} k_{\gamma}}$ normalized to the cross section $\sigma$.

An analysis of the general expression (6) shows that variation of the direction of the atomic polarization affects only those spherical harmonics $Y_{k_{0} q_{0}}\left(\theta_{a}, \phi_{a}\right)$ with $k_{0}>0$ in the geometrical factor $F_{k_{0} k_{\gamma}}$ [Eqs. (3) and (4)]. All other factors remain unchanged. Therefore, any dichroism (the difference of cross sections for two directions of the atomic polarization) is described by terms with $k_{0}>0$. Furthermore, only terms proportional to odd statistical tensors $\mathcal{A}_{10}, \mathcal{A}_{30}, \ldots$ contribute to the CMD and LMDAD, i.e., magnetic dichroism is determined by the atomic orientation. On the other hand, only terms proportional to even statistical tensors $\mathcal{A}_{20}, \mathcal{A}_{40}, \ldots$ contribute to LAD, i.e., the linear alignment dichroism is determined by the atomic alignment.

We will now rewrite Eq. (5) in the nonrelativistic approximation. We assume that both initial and final atomic states are well described in the $L S J$ coupling approximation, i.e., the spin-orbit effects are neglected except for the finestructure splitting. Besides, we suppose that the wave function of the photoelectron does not depend on $j$. Therefore, the dynamics of the process does not depend on the values of the total angular momenta $J, J_{0}, J_{f}, j$. It should, however, be noted that this assumption does not hold in the region of autoionizing resonances; an example will be considered in Sec. IV B. Deviations from this approximation have also been reported for excitation energies above, but close to threshold [20,21]. In the present paper, except for Sec. IV B, we assume that the photon energy is chosen to be sufficiently far above threshold that such effects are excluded. In the $L S J$ coupling approximation each dipole amplitude appearing in Eq. (5) can be presented as a combination of the reduced dipole amplitudes for corresponding $L S$ states according to

$$
\begin{aligned}
& \left\langle\alpha_{f} L_{f} S_{f} J_{f}, l j: J\|D\| \alpha_{0} L_{0} S_{0} J_{0}\right\rangle \\
& =\hat{J}_{0} \hat{J}_{0} \hat{J}_{f} \hat{j} \sum_{L S}(-1)^{J_{0}+S_{0}+L+1} \hat{L}\left\{\begin{array}{ccc}
L & J & S_{0} \\
J_{0} & L_{0} & 1
\end{array}\right\} \\
& \quad \times\left\{\begin{array}{ccc}
L_{f} & l & L \\
S_{f} & \frac{1}{2} & S \\
J_{f} & j & J
\end{array}\right\} D_{l L S} \delta_{S S_{0}},
\end{aligned}
$$

where $L_{0} S_{0}$ and $L_{f} S_{f}$ are the orbital angular momenta and spins of the initial atomic and final ionic states, respectively; $\delta_{S S_{0}}$ is a Kronecker symbol and

$$
D_{l L S}=\left\langle\alpha_{f} L_{f} S_{f}, l \frac{1}{2}: L S\|D\| \alpha_{0} L_{0} S_{0}\right\rangle
$$

is the multichannel dipole matrix element in the $L S$ approximation with the asymptotic channels characterized by the quantum numbers $\alpha_{f}, L_{f}, S_{f}, l, L, S$. The dipole operator does not operate on spin variables, therefore, $S=S_{0}$. In general the matrix element in Eq. (11) depends on the initial and final spins $S_{0}, S_{f}$ due to, for example, exchange interaction or through the parentage. It follows from the $9 j$ symbol in Eq. (10) that in the LSJ coupling approximation, the photoionization transitions are possible only if the spins satisfy the triangle rule $\vec{S}_{f}+\vec{S}_{0}+\frac{\overrightarrow{1}}{2}=0$.

After the substitution of Eq. (10) into Eq. (5), the summation over the angular momenta $j, j^{\prime}, J, J^{\prime}$, appearing in Eq. (5) can be performed analytically by making use of standard expressions of angular-momentum theory [22]. The result can be cast into the form

$$
\begin{aligned}
& B_{k_{0} k k_{\gamma}}=3 \hat{J}_{0}^{3} \hat{J}_{f}^{2}(-1)^{J_{f}+L_{f}-S_{0}-1 / 2+k_{0}} \\
& \times \sum_{x_{0} x_{f} x} \hat{x}_{0}^{2} \hat{x}_{f}^{2} \hat{x}^{2}(-1)^{x_{f}+x}\left\{\begin{array}{lll}
S_{f} & S_{f} & x_{f} \\
L_{f} & L_{f} & J_{f}
\end{array}\right\} \\
& \times\left\{\begin{array}{ccc}
S_{f} & S_{f} & x_{f} \\
S_{0} & S_{0} & \frac{1}{2}
\end{array}\right\}\left\{\begin{array}{ccc}
k_{0} & k & k_{\gamma} \\
x & x_{0} & x_{f}
\end{array}\right\}\left\{\begin{array}{ccc}
L_{0} & S_{0} & J_{0} \\
L_{0} & S_{0} & J_{0} \\
x_{0} & x_{f} & k_{0}
\end{array}\right\} \\
& \times \sum_{l l^{\prime} L L^{\prime}}(-1)^{l} \hat{L} \hat{L}^{\prime} \hat{l} \hat{l}^{\prime}\left(l 0 l^{\prime} 0 \mid k 0\right) \\
& \times\left\{\begin{array}{ccc}
L_{0} & 1 & L \\
L_{0} & 1 & L^{\prime} \\
x_{0} & k_{\gamma} & x
\end{array}\right\}\left\{\begin{array}{ccc}
L_{f} & l & L \\
L_{f} & l^{\prime} & L^{\prime} \\
x_{f} & k & x
\end{array}\right\} D_{l L S_{0}} D_{l^{\prime} L^{\prime} S_{0}}^{*}
\end{aligned}
$$

An equivalent expression in the $L S J$ approximation was obtained by Cherepkov et al. [23,2]; however, in Eq. (12) we have kept the multielectron matrix elements in the $L S$ approximation while Cherepkov et al. reduce them to singleelectron matrix elements.

The monopole coefficient $B_{000}$ related to the angleintegrated cross section $\sigma$ is obtained from Eq. (12): 


$$
\begin{aligned}
B_{000}= & \sqrt{3} \hat{J}_{0}^{2} \hat{J}_{f}^{2}(-1)^{J_{f}+J_{0}+1 / 2} \sum_{x}(-1)^{x} \hat{x}^{2} \\
& \times\left\{\begin{array}{ccc}
L_{0} & L_{0} & x \\
S_{0} & S_{0} & J_{0}
\end{array}\right\}\left\{\begin{array}{ccc}
S_{0} & S_{0} & x \\
S_{f} & S_{f} & \frac{1}{2}
\end{array}\right\}\left\{\begin{array}{lll}
S_{f} & S_{f} & x \\
L_{f} & L_{f} & J_{f}
\end{array}\right\} \\
& \times \sum_{l L L^{\prime}}(-1)^{l} \hat{L} \hat{L}^{\prime}\left\{\begin{array}{ccc}
L_{f} & L_{f} & x \\
L & L^{\prime} & l
\end{array}\right\} \\
& \times\left\{\begin{array}{ccc}
L & L^{\prime} & x \\
L_{0} & L_{0} & 1
\end{array}\right\} D_{l L S_{0}} D_{l L^{\prime} S_{0}}^{*} .
\end{aligned}
$$

Equation (13), together with Eqs. (7) and (9), are equivalent to Eqs. (7) and (8) of Ref. [24] and describe the atomic photoionization cross section in the case of fine-structure resolved atomic and ionic states. From Eq. (13) it can be seen that, in general, the cross section $\sigma$ does not reduce to the sum of absolute squares of the amplitudes but depends on the interference between ionization channels with different total orbital momenta $L$ [25]. Furthermore, the cross section $\sigma$ displays a nonstatistical behavior with respect to the fine structure $J_{f}$ of the residual ion.

\section{Atoms with S symmetry}

The coefficients $B_{k_{0} k k_{\gamma}}$ given by Eq. (12) can be considerably simplified for cases where the orbital angular momentum vanishes in the initial state $\left(L_{0}=0\right)$. This case includes, in particular, atoms with semifilled subshells, such as $\mathrm{Cr}$ $3 d^{5} 4 s^{7} S_{3}[26,13,27]$ and $\mathrm{Eu} 4 f^{7} 6 s^{28} S_{7 / 2}[28,29,6]$; these examples will be discussed in Sec. IV. After the substitution of $L_{0}=0$ into Eq. (12) we have $L=L^{\prime}=1$, and performing the sum over $x_{0}, x_{f}, x$ we obtain

$$
\begin{aligned}
B_{k_{0} k k_{\gamma}}^{S}= & C_{k_{0}}^{S}\left(J_{0}, J_{f}\right) \sum_{l l^{\prime}}(-1)^{l} \hat{l} \hat{l}^{\prime}\left(l 0, l^{\prime} 0 \mid k 0\right) \\
& \times\left\{\begin{array}{ccc}
L_{f} & l & 1 \\
L_{f} & l^{\prime} & 1 \\
k_{0} & k & k_{\gamma}
\end{array}\right\} D_{l 1 S_{0}} D_{l^{\prime} 1 S_{0}}^{*},
\end{aligned}
$$

where

$$
\begin{aligned}
C_{k_{0}}^{S}\left(J_{0}, J_{f}\right)= & 3 \hat{S}_{0} \hat{J}_{f}^{2}(-1)^{k_{0}+L_{f}+J_{f}-J_{0}-1 / 2} \\
& \times\left\{\begin{array}{lll}
S_{f} & S_{f} & k_{0} \\
L_{f} & L_{f} & J_{f}
\end{array}\right\}\left\{\begin{array}{ccc}
S_{f} & S_{f} & k_{0} \\
S_{0} & S_{0} & \frac{1}{2}
\end{array}\right\} .
\end{aligned}
$$

The superscript $S$ here and below refers to atoms with $S$ symmetry $\left(L_{0}=0\right)$. As an important result, the coefficients (14) are written as a product of (i) an angular-momentum coupling factor $C_{k_{0}}^{S}\left(J_{0}, J_{f}\right)$, Eq. (15), which determines the dependence on the total angular momenta $J_{0}, J_{f}$ in the initial atomic and final ionic states, and (ii) a dynamical part including the dipole matrix elements, which does not depend on these quantum numbers and is thus constant for each multiplet in the final ionic state. We want to stress that Eq. (14) contains the full multichannel dipole amplitudes which include various kinds of many-electron correlations. The monopole coefficient $B_{000}$ for atoms with $S$ symmetry can be obtained either from Eq. (13) or from Eqs. (14) and (15):

$$
B_{000}^{S}=\sqrt{3} \frac{\left(2 J_{f}+1\right)}{\left(2 L_{f}+1\right)\left(2 S_{f}+1\right)} \sum_{l}\left|D_{l 1 S_{0}}\right|^{2} .
$$

For atoms with $S$ symmetry, the cross section $\sigma$ displays a statistical behavior with respect to the fine structure $J_{f}$ of the photoion.

\section{B. Single-configuration approximation}

In many cases the theoretical description is further simplified by using the single-configuration approximation. We assume that the initial atomic state is well described by a single electronic configuration, and, furthermore, take into account only that final ionic configuration, which results from the initial atomic configuration by removing a single electron from the bound orbital $n_{0} l_{0}$. Besides, we suppose that the single-electron wave functions in the initial and final states, including the continuum electron wave function are independent of the many-electron coupling (quantum numbers $L_{0}, S_{0}, L_{f}, S_{f}, L, S$ ); for example, they may be solutions of the configuration-averaged Hartree-Fock (HF) equations. Within the above approximations the manyelectron dipole amplitudes $D_{l L S}$ can be reduced to the oneelectron dipole amplitudes $D_{l}$ using the Racah algebra, as has been shown elsewhere [30,31]:

$$
\begin{gathered}
D_{l L S}=G(L S) D_{l} K, \\
D_{l} \equiv\left\langle\varepsilon l\|d\| n_{0} l_{0}\right\rangle=i^{-l} e^{i \delta_{l}}\left|D_{l}\right| .
\end{gathered}
$$

Here $G(L S)$ is a factor which contains the angularmomentum coupling as well as the fractional parentage coefficients; it depends on the shell structure of the target atom. $D_{l}$ is a complex dipole amplitude of a single-electron transition from a bound orbital $n_{0} l_{0}$ to the continuum $\varepsilon l$. The reduction (17) leads to a conventional model of photoionization with three dynamical parameters: two absolute values of the dipole amplitudes $D_{l}$ and a corresponding phase difference. The overlap integral $K$ takes into account, as a first approximation, the relaxation of the atomic core; $K$ is unity in the frozen-core approximation. According to the approximations introduced above, $K$ is independent of the atomic and ionic orbital angular momenta and spins. The general conclusions drawn in the following do not depend on the overlap integral that will be omitted in the following expressions.

As a consequence of the reduction equation (17), the summation over $L, L^{\prime}$ in Eq. (12) can be performed analytically, and the coefficients $B_{k_{0} k k_{\gamma}}$ can be further reduced to a product of two factors:

$$
B_{k_{0} k k_{\gamma}}=C_{k_{0}}\left(L_{f}, S_{f}, J_{f}\right) b_{k_{0} k k_{\gamma}} .
$$

The coefficients $C_{k_{0}}\left(L_{f}, S_{f}, J_{f}\right)$ contain all many-electron angular-momentum coupling coefficients and are obtained from Eq. (12) and the coupling factors $G(L S)$ in Eq. (17); examples will be given below. The dependence on the angular momenta, $L_{f}, S_{f}, J_{f}$ of the photoion is completely sepa- 
rated from the dynamics of the photoionization process; the latter is determined by the one-electron parameters

$$
b_{k_{0} k k_{\gamma}}=\sum_{l l^{\prime}}(-1)^{l} \hat{l} \hat{l}^{\prime}\left(l 0, l^{\prime} 0 \mid k 0\right)\left\{\begin{array}{ccc}
l_{0} & l & 1 \\
l_{0} & l^{\prime} & 1 \\
k_{0} & k & k_{\gamma}
\end{array}\right\} D_{l} D_{l^{\prime}}^{*} .
$$

A triangle rule in the $9 j$ symbol requires $k_{0} \leqslant 2 l_{0}$ which implies that, within our approximations, no dichroism can be measured in the emission of an $s$ electron $\left(l_{0}=0\right)$. This constraint is also very helpful in other cases; for example, in the emission of a $p$ electron only the lowest statistical tensors $\mathcal{A}_{10}, \mathcal{A}_{20}$ contribute to dichroism within our approximations. The neglect of higher terms was introduced ad hoc in a number of previous publications $[26,13,27]$; in the present treatment, it is a consequence of the approximations made (this result was obtained in Ref. [2]).

It follows from Eqs. (8) and (19) that the generalized anisotropy parameters $\beta_{k_{0} k k_{\gamma}}$ can also be written in a product form $^{2}$ :

$$
\beta_{k_{0} k k_{\gamma}}=\sqrt{3} \frac{C_{k_{0}}\left(L_{f}, S_{f}, J_{f}\right)}{C_{0}\left(L_{f}, S_{f}, J_{f}\right)} \frac{b_{k_{0} k k_{\gamma}}}{b_{000}} .
$$

In the energy range far from resonances the dipole amplitudes $D_{l}$ (and thus the factors $b_{k_{0} k_{\gamma}}$ ) are slowly varying functions of the photoelectron energy. Therefore, the parameters $b_{k_{0} k k_{\gamma}}$ can be considered as approximately constant over the range of a multiplet or even of several neighboring multiplets. An important consequence of this is that, within the given approximations, the relative strength of dichroism in the photoelectron spectrum is independent of the dynamics of the photoionization process, and is in fact determined by the angular-momentum coupling coefficients $C_{k_{0}}\left(L_{f}, S_{f}, J_{f}\right)$. One has to keep in mind, however, that according to Eqs. (2) and (19) the absolute scale, and also the overall sign, of the dichroism spectrum are both determined by the dynamical coefficients $b_{k_{0} k_{\gamma}}$ by the magnitude of the atomic polarization $\mathcal{A}_{k_{0} 0}$, and by the particular geometry represented by the geometrical factors $F_{k_{0} k_{\gamma}}$. Furthermore, the relative contribution from terms with different $k_{0}$ (i.e., from higher state multipoles of the target atom) to the dichroism spectrum still depends on the dynamics of photoionization. This can be seen from Eq. (19) where the coupling coefficients $C_{k_{0}}\left(L_{f}, S_{f}, J_{f}\right)$ depend on the rank $k_{0}$.

In the following sections the coefficients $C_{k_{0}}$ are derived for a number of practically important cases. Atoms with vanishing orbital angular momentum $\left(L_{0}=0\right)$ are considered shortly before turning to the general case $L_{0} \geqslant 0$.

\section{Atoms with $S$ symmetry}

The dichroism in the photoelectron spectra of atoms with $S$ symmetry can be described within the general frame of

\footnotetext{
${ }^{2}$ The product form of a particular $\beta$ coefficient, which determines the magnetic dichroism, was obtained for atoms with $J_{0}=\frac{1}{2}$ in [5].
}

Sec. II A [Eqs. (14) and (15)] once the symmetries of the multiplets in the photoelectron spectrum are known. For the sake of completeness we also consider the description in the single-configuration approximation here.

For $L_{0}=0$, the reduction equation (17) of the dipole matrix elements become

$$
D_{l L S}=Q D_{l} \delta_{L_{f} l_{0}} \delta_{S S_{0}}
$$

where $Q$ is a factor depending, in general, on the initial atomic configuration; its explicit form is not relevant for the further discussion. Inserting Eq. (22) into Eq. (14) immediately leads to the product form Eq. (19) with coupling coefficients $C_{k_{0}}\left(L_{f}, S_{f}, J_{f}\right)$,

$$
C_{k_{0}}^{S}\left(L_{f}, S_{f}, J_{f}\right)=Q^{2} C_{k_{0}}^{S}\left(J_{0}, J_{f}\right) \delta_{l_{0} L_{f}}
$$

the coefficients $C_{k_{0}}^{S}\left(J_{0}, J_{f}\right)$ on the right side are given by Eq. (15).

\section{Atoms with $P, D, F, \ldots$ symmetry}

In the general case $L_{0} \geqslant 0$ the product form Eq. (19) can be obtained in a way similar to that followed above. The discussion will be split to cover two practically important cases: (i) ionization of a closed subshell (which applies, for example, to the $3 p$ shell of the $3 d$ transition metal atoms and the $4 d$ shell of the rare earth atoms) and (ii) ionization of an open subshell (for example, the $3 d$ shell of the $3 d$ transition metal atoms or the $4 f$ shell of the rare earth atoms).

First we consider photoionization of an inner closed subshell $n_{0} l_{0}$ of an atom that has only one open shell $\tilde{n} \widetilde{l}$ in the initial state:

$$
\begin{aligned}
& \cdots\left(n_{0} l_{0}\right)^{2\left(2 l_{0}+1\right)} \cdots(\tilde{n} \widetilde{l})^{q}+\gamma \\
& \rightarrow \cdots\left(n_{0} l_{0}\right)^{2\left(2 l_{0}+1\right)-1} \cdots(\tilde{n} \widetilde{l})^{q}+\varepsilon l,
\end{aligned}
$$

where the dots indicate closed subshells common to initial and final atomic/ionic configurations. Within the approximations discussed above (Sec. II B), the reduction equation (17) of the dipole matrix elements in this case reads

$$
\begin{aligned}
& D_{l L S}=(-1)^{q+l+L+L_{f}} \hat{L}_{f} \hat{L} \hat{S}_{f} \hat{S}_{0}^{-1} \\
& \times\left\{\frac{1}{2} S_{f} S_{0}\right\}\left\{\begin{array}{ccc}
L_{0} & L_{f} & l_{0} \\
l & 1 & L
\end{array}\right\} D_{l} \delta_{S S_{0}},
\end{aligned}
$$

where $\left\{\frac{1}{2} S_{f} S_{0}\right\}$ is a $3 j$ symbol that is unity if the triangle condition $\vec{S}_{f}+\vec{S}_{0}+\frac{\vec{r}}{2}=0$ is fulfilled and vanishes otherwise. By inserting Eq. (25) into Eq. (12), the summation over $L, L^{\prime}, x_{0}, x_{f}$ can now be performed; the final result is of the form of Eqs. (19) and (20) with coupling factors 


$$
\begin{aligned}
C_{k_{0}}^{\text {closed }}\left(L_{f}, S_{f}, J_{f}\right)= & 3 \hat{J}_{0}^{3} \hat{S}_{0}^{-2} \hat{S}_{f}^{2} \hat{J}_{f}^{2} \hat{L}_{f}^{2} \\
& \times \sum_{x}(-1)^{J_{0}+x+l_{0}} \hat{x}^{2}\left\{\begin{array}{ccc}
J_{0} & J_{0} & k_{0} \\
l_{0} & l_{0} & x
\end{array}\right\} \\
& \times\left\{\begin{array}{ccc}
J_{f} & L_{f} & S_{f} \\
S_{0} & \frac{1}{2} & x
\end{array}\right\}^{2}\left\{\begin{array}{ccc}
J_{0} & S_{0} & L_{0} \\
L_{f} & l_{0} & x
\end{array}\right\}^{2} .
\end{aligned}
$$

The summation over $x$ in Eq. (26) runs over not more than two arguments $J_{f} \pm \frac{1}{2}$. Since the reduced anisotropy coefficients $b_{k_{0} k k_{\gamma}}$ [Eq. (20)], in general, contain a maximum of three terms, it is clear from Eq. (19) that every dynamical coefficient $B_{k_{0} k k_{\gamma}}$ consists of not more than six terms; this means a considerable simplification for practical purposes in comparison with the general formulation of the parameters $B_{k_{0} k_{\gamma}}$ [Eq. (12)], which contains orders of magnitudes more terms. For the generalized anisotropy parameters [Eq. (8)], the monopole coefficient $B_{000}$ is needed; it is given by

$$
\begin{aligned}
B_{000}^{\text {closed }}= & \sqrt{3} \hat{J}_{0}^{2} \hat{S}_{0}^{-2} \hat{S}_{f}^{2} \hat{J}_{f}^{2} \hat{L}_{f}^{2} \hat{l}_{0}^{-2} \\
& \times \sum_{j_{0}} \hat{j}_{0}^{2}\left\{\begin{array}{ccc}
\frac{1}{2} & l_{0} & j_{0} \\
S_{0} & L_{0} & J_{0} \\
S_{f} & L_{f} & J_{f}
\end{array}\right\}^{2} \sum_{l}\left|D_{l}\right|^{2}
\end{aligned}
$$

in agreement with Eq. (10.1) of Ref. [32].

The second case of general interest concerns photoionization of an inner open subshell $n_{0} l_{0}$ of an atom with all other subshells closed:

$$
\cdots\left(n_{0} l_{0}\right)^{q \cdots+\gamma \rightarrow \cdots\left(n_{0} l_{0}\right)^{q-1} \cdots+\varepsilon l .}
$$

In close similarity to Eq. (25) the dipole amplitudes can be reduced to the one-electron amplitudes. In this case one obtains the coupling factors $C_{k_{0}}$ :

$$
C_{k_{0}}^{\text {open }}=q \hat{L}_{0}^{2} \hat{S}_{0}^{2} \hat{L}_{f}^{-2} \hat{S}_{f}^{-2}\left(l_{0}^{q} L_{0} S_{0}\left\{\mid l_{0}^{q-1} L_{f} S_{f}\right)^{2} C_{k_{0}}^{\text {closed }},\right.
$$

where the quantities in parenthesis are the coefficients of fractional parentage and $C_{k_{0}}^{\text {closed }}$ is given by Eq. (26). The monopole coefficient $B_{000}$ reads in complete analogy

$$
B_{000}^{\text {open }}=q \hat{L}_{0}^{2} \hat{S}_{0}^{2} \hat{L}_{f}^{-2} \hat{S}_{f}^{-2}\left(l_{0}^{q} L_{0} S_{0}\left\{\mid l_{0}^{q-1} L_{f} S_{f}\right)^{2} B_{000}^{\text {closed }},\right.
$$

with $B_{000}^{\text {closed }}$ given by Eq. (27).

\section{IMPLICATIONS FOR THE DICHROISM IN PHOTOELECTRON SPECTRA}

In the present section general conclusions on the spectral dependence of dichroism will be drawn solely from the analysis of the angular-momentum coupling factors $C_{k_{0}}$ introduced in the previous section.

\section{A. Sum rules}

Here we consider the integration of the dichroism in the photoelectron spectrum over the energy range of one or more final ionic multiplets. Summation of the dynamical coefficients [Eq. (12)] over the fine structure levels $J_{f}$ of a finalstate multiplet $L_{f} S_{f}$ can easily be performed using the relation $[22]$

$$
\sum_{J_{f}}(-1)^{L_{f}+S_{f}+J_{f} \hat{J}_{f}^{2}}\left\{\begin{array}{lll}
S_{f} & S_{f} & x_{f} \\
L_{f} & L_{f} & J_{f}
\end{array}\right\}=\hat{L}_{f} \hat{S}_{f} \delta_{x_{f}} 0,
$$

which gives (after additional summation over $x_{0}, x_{f}, x$ )

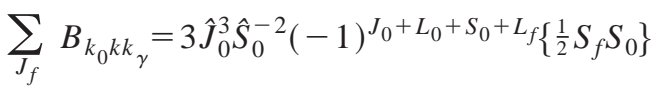

$$
\begin{aligned}
& \times\left\{\begin{array}{lll}
J_{0} & J_{0} & k_{0} \\
L_{0} & L_{0} & S_{0}
\end{array}\right\} \\
& \times \sum_{l l^{\prime} L L^{\prime}}(-1)^{L^{\prime}} \hat{L} \hat{L}^{\prime} \hat{l}^{\prime} \hat{l}^{\prime}\left(l 0, l^{\prime} 0 \mid k 0\right) \\
& \times\left\{\begin{array}{ccc}
L & l & L_{f} \\
l^{\prime} & L^{\prime} & k
\end{array}\right\}\left\{\begin{array}{ccc}
L_{0} & L & 1 \\
L_{0} & L^{\prime} & 1 \\
k_{0} & k & k_{\gamma}
\end{array}\right\} \\
& \times D_{l L S_{0}} D_{l^{\prime} L^{\prime} S_{0}}^{*} .
\end{aligned}
$$

\section{Atoms with $S$ symmetry}

For $L_{0}=0$, the triangle rule $k_{0} \leqslant 2 L_{0}$ for the $6 j$ and $9 j$ symbols in Eq. (32) implies that $k_{0}=0$. Since dichroism is related to terms with $k_{0}>0$, we immediately arrive at the following sum rule for atoms with $S$ symmetry:

Sum rule 1. In the nonrelativistic approximation, the dichroism in the photoelectron spectra of atoms with zero orbital angular momentum in the initial state strictly vanishes for each final ionic multiplet characterized by $L_{f}$ and $S_{f}$ when being integrated over the fine structure $J_{f}$.

This is, in fact, a very general result since it is based on the expression (12) valid if the spin-orbit interaction is negligible. All kinds of many-electron correlations, e.g., configuration interaction can be included in the calculations of the dipole amplitudes; the sum rule remains valid.

The sum rule 1 has a simple physical explanation. It is clear from the vector model of $L S J$ coupling that summation over $J_{f}$ is equivalent to the integration over all spin directions $\vec{S}_{f}$ of the photoion. Taking into account that the spin of the photoelectron is not detected and that the dipole interaction is spin independent, it can be concluded that the spin orientation cannot influence the process and that the spin can in fact be disregarded. Therefore, the dichroism can only be connected with the polarization of the initial orbital angular momentum $L_{0}$, which vanishes in the case considered; consequently, there is no dichroism.

Since the fine-structure splitting is reflected in the kinetic energy $E_{\text {kin }}$ of the photoelectron, the sum rule can also be formulated as 


$$
\int d E_{\mathrm{kin}}\left[\left(\frac{d \sigma}{d \Omega}\right)_{\vec{A}}-\left(\frac{d \sigma}{d \Omega}\right)_{\vec{A}^{\prime}}\right]=0,
$$

where $\vec{A}$ and $\vec{A}^{\prime}$ correspond to two arbitrary directions of the atomic polarization, and the integration is performed over the energy range of the photoelectron lines corresponding to an ionic multiplet $L_{f}, S_{f}$.

\section{Atoms with $P, D, F, \ldots$ symmetry}

Equation (32) shows that, for atoms with $L_{0}>0$, the sum $\Sigma_{J_{f}} B_{k_{0} k k_{\gamma}}$ is generally nonzero for $k_{0}>0$; therefore, the dichroism integrated over the fine structure of a single photoelectron multiplet line does not vanish. However, another approximate sum rule is valid in this case for the ionization of a closed subshell in the single-configuration approximation (see Sec. II B). Performing the summation over $J_{f}$ in Eq. (26), we obtain

$$
\begin{aligned}
D_{k_{0}}\left(L_{f}\right)= & \sum_{J_{f}} C_{k_{0}}^{\text {closed }} \\
= & 3 \hat{J}_{0}^{3} \hat{S}_{0}^{-4} \hat{L}_{f}^{2} \hat{S}_{f}^{2}(-1)^{k_{0}+L_{f}+J_{0}+S_{0}+l_{0}} \\
& \times\left\{\begin{array}{lll}
J_{0} & J_{0} & k_{0} \\
L_{0} & L_{0} & S_{0}
\end{array}\right\}\left\{\begin{array}{ccc}
L_{0} & L_{0} & k_{0} \\
l_{0} & l_{0} & L_{f}
\end{array}\right\}\left\{\frac{1}{2} S_{f} S_{0}\right\},
\end{aligned}
$$

where the fine-structure integrated quantities $D_{k_{0}}$ have been introduced, which are convenient for further discussion. As an important consequence, the dependence of the coefficients $D_{k_{0}}$ on the orbital angular momentum $L_{f}$ is formally equivalent to the dependence of the coefficients $C_{k_{0}}^{S}$ [Eq. (15)] on the total angular momentum $J_{f}$. It follows immediately that another (but less general) sum rule holds in the present case. To be more specific, since we assumed that the ionic and photoelectron orbitals do not depend on $L_{f}$, one can perform the summation of the coefficients $D_{k_{0}}$ [Eq. (34)] over $L_{f}$ using an expression similar to Eq. (31). The result again vanishes for $k_{0}>0$, which gives the following sum rule for atoms with $P, D, F, \ldots$ symmetry.

Sum rule 2. In the nonrelativistic single-configuration approximation (see Sec. II B), the dichroism in the ionization of an inner closed subshell strictly vanishes separately for both the high-spin $\left(S_{f}=S_{0}+\frac{1}{2}\right)$ and low-spin $\left(S_{f}=S_{0}-\frac{1}{2}\right)$ parts of the photoelectron spectrum associated with the vacancy $\left(n_{0} l_{0}\right)^{-1}$ when being integrated over the term structure of the final ionic state corresponding to different angular momenta $L_{f}$.

The separate validity for the high-spin and the low-spin spectrum follows since the coefficients $D_{k_{0}}$ [Eq. (34)] do not depend on the final ionic spin $S_{f}$. This sum rule can also be expressed in the integral from of Eq. (33) if the integration is performed over the somewhat larger energy range due to the electrostatic and exchange splittings. Both sum rules 1 and 2 should also provide a helpful consistency check for practical calculations.

The sum rules in this paper should not be confused with those obtained by Thole and co-workers [33,34], which re- late the circular dichroism integrated over an absorption edge, i.e., along the photon energy axis, to ground-state magnetic moments. Thole and van der Laan $[35,36]$ have also considered the integration of dichroism over the photoelectron spectrum. Their arguments concerning the ionization of closed subshells [[35], first section and [36] Eq. (2)], however, are based on a single-electron picture and are therefore not valid for open-shell atoms in cases when intershell coupling is strong. Our sum rule 1 for atoms with $S$ symmetry has been derived under very general assumptions; Thole and van der Laan do not go beyond the single-configuration approximation.

\section{B. Spectral patterns of dichroism in photoelectron spectra}

While in Sec. III A the dichroism was analyzed with respect to integration over the kinetic energy of the photoelectron, in this section the kinetic-energy dependence is considered. Our aim is to establish typical patterns common to the spectra of different atoms and for different types of nonresonant photoionization processes.

\section{Atoms with $S$ symmetry}

In considering atoms with $S$ symmetry it is necessary to study the explicit dependence of the coupling factors $C_{k_{0}}^{S}$ [Eq. (15)] on the total angular momentum $J_{f}$; this dependence is determined by a $6 j$ symbol, which has a simple structure since it contains two pairs of identical coefficients. The factors $C_{k_{0}}^{S}$ reduce to a polynomial of the degree $2 k_{0}$ in $J_{f}$ since the relevant $6 j$ symbol can be expressed as [22]

$$
\begin{aligned}
\left\{\begin{array}{ccc}
S_{f} & S_{f} & k_{0} \\
L_{f} & L_{f} & J_{f}
\end{array}\right\}= & (-1)^{L_{f}+S_{f}+J_{f}+k_{0}} \\
& \times\left[\frac{\left(2 S_{f}-k_{0}\right) !\left(2 L_{f}-k_{0}\right) !}{\left(2 S_{f}+k_{0}+1\right) !\left(2 L_{f}+k_{0}+1\right) !}\right]^{1 / 2} \\
& \times V_{k_{0}}(x)
\end{aligned}
$$

where $V_{k_{0}}(x)$ is a polynomial of the degree $k_{0}$ in the variable

$$
x=J_{f}\left(J_{f}+1\right)-L_{f}\left(L_{f}+1\right)-S_{f}\left(S_{f}+1\right) .
$$

Since the variable $x$ is quadratic in $J_{f}$, each root of $V_{k_{0}}(x)$ is related to two values of $J_{f}$; however, one of these values is always negative and thus not meaningful. The other value of $J_{f}$ lies within the allowed interval $\left|L_{f}-S_{f}\right|, \ldots, L_{f}+S_{f}$. The fine-structure components are arranged in a regular (assending or descending) way on the energy axis due to the Landé interval rule. Now, since the degree $k_{0}$ of the polynomials $V_{k_{0}}$ is identical with the rank of the statistical tensors $\mathcal{A}_{k_{0} 0}$, we obtain the following result:

Spectral patterns of dichroism for atoms with S symmetry. The contribution of each specific statistical tensor $\mathcal{A}_{k_{0} 0}$ of the atomic polarization to the dichroism in a photoelectron line corresponding to a photoion multiplet of definite symmetry $L_{f}, S_{f}$ shows a characteristic pattern as a function of the fine structure $J_{f}$. In particular, the number of zeros of dichroism within each photoelectron line is given by the rank $k_{0}$ of the respective statistical tensor. 
LMDAD / CMD

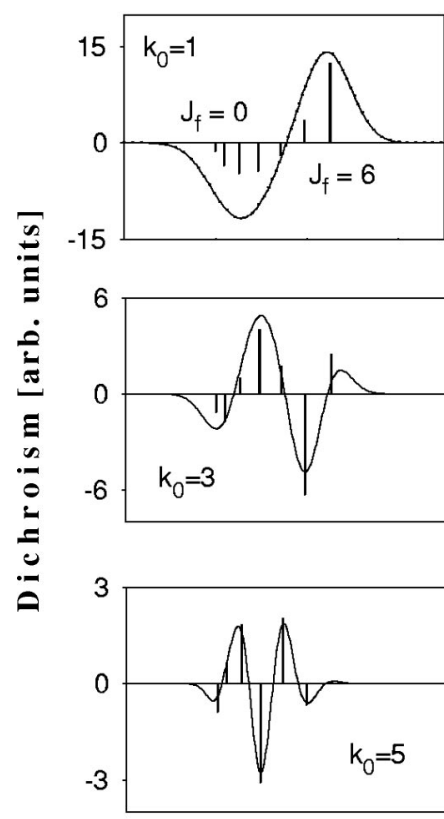

LAD
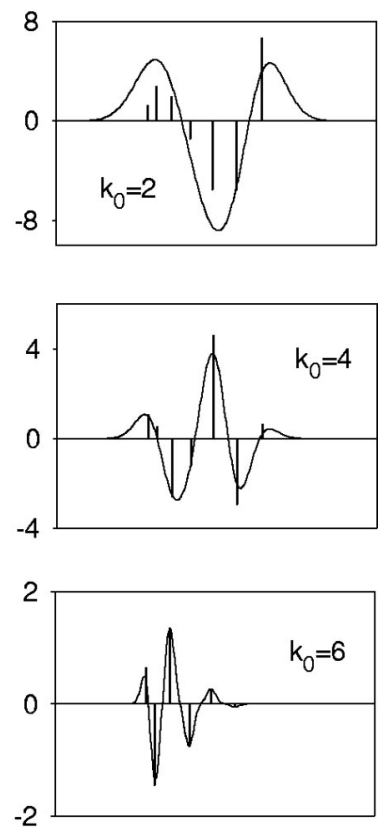

Energy

FIG. 2. Spectral patterns of magnetic dichroism (LMDAD/ CMD) and linear alignment dichroism (LAD) for atoms with $S$ symmetry in the $L S J$ coupling approximation for a ${ }^{7} F$ final ionic state. For the solid line, a Gaussian broadening of arbitrary width (decreasing with $k_{0}$ ) was assumed in order to stress the specific character of the respective pattern.

This result is independent of any specific experimental geometry. It is rather general since it is based only on the nonrelativistic approximation which has led to Eq. (15). In particular, the appearance of spectral patterns is independent of the specific symmetry $L_{f}, S_{f}$ of a photoelectron line, of the number of fine-structure components, and of the relative contribution of the partial photoionization amplitudes and their phase difference.

For illustration we consider first LMDAD and CMD. These are related to the odd statistical tensors $\mathcal{A}_{10}, \mathcal{A}_{30}, \mathcal{A}_{50}, \ldots$, and the corresponding spectral patterns of the order 1, 3, 5 are depicted on the left-hand side of Fig. 2. For Fig. 2 we have chosen $S_{0}=\frac{7}{2}, L_{f}=S_{f}=3$, but due to their general nature, the patterns are similar for any other quantum numbers. The height of the bars, which are arranged on the energy axis according to the Lande interval rule, is given by the value of the coefficients $C_{k_{0}}^{S}\left(\frac{7}{2}, J_{f}\right)$ of Eq. (15) and, therefore, reflects the polynomials $V_{k_{0}}$ at the discrete positions $J_{f}=0,1, \ldots, 6$. For the dominating contribution from the atomic orientation $\left(k_{0}=1\right)$, the polynomial and the root are given by

$$
\begin{gathered}
V_{1}(x)=-2 x \\
\operatorname{root}: J_{f}=\left[L_{f}\left(L_{f}+1\right)+S_{f}\left(S_{f}+1\right)+\frac{1}{4}\right]^{1 / 2}-\frac{1}{2} .
\end{gathered}
$$

The solid line is obtained from the bar spectrum by assuming a Gaussian broadening; the width was chosen arbitrarily in order to pronounce the respective pattern. The contribution of higher moments $\mathcal{A}_{30}, \mathcal{A}_{50}, \ldots$, compared to that of the dominating multipole $\mathcal{A}_{10}$, depends on the dynamics of photoionization, as well as on the experimental geometry. As experiments show $[13,28,29]$, the contribution of higher multipoles can be neglected in many cases. For photoionization of a $p$ electron there are no contributions from multipoles with $k_{0}>2$, as has been noted earlier. In general, in order to observe the influence of higher state multipoles one should (i) choose a special geometry with the detector set at a minimum of the angular distribution of the leading multipoles [42] and (ii) perform fine-structure resolved measurements since the higher-order dichroism patterns display more lobes (see Fig. 2) and therefore are leveled more strongly than the leading ones if the instrumental bandpass is larger than the fine-structure splitting.

Following these considerations, the dominating contribution to LMDAD and CMD is most often well described by the coupling coefficients $C_{1}^{S}$. The spectrum on the top of the left-hand side of Fig. 2 therefore shows the typical pattern of magnetic dichroism (LMDAD / CMD) with two characteristic lobes of opposite sign and one central node.

Similar considerations apply to the LAD related to the even statistical tensors $\mathcal{A}_{20}, \mathcal{A}_{40}, \ldots$. The spectral patterns of the order 2, 4, 6 are depicted on the right-hand side of Fig. 2. LAD is usually dominated by the contribution of the atomic alignment $\mathcal{A}_{20}$ and is therefore described by the coupling coefficients $C_{2}^{S}$. The typical spectral pattern of LAD is given by the spectrum on the top of the right-hand side of Fig. 2. It shows three lobes of alternating sign and two nodes. The polynomial and the roots of second order are

$$
\begin{aligned}
V_{2}(x)= & 6 x^{2}+6 x-8 S_{f} L_{f}\left(S_{f}+1\right)\left(L_{f}+1\right), \\
\text { roots: } J_{f}= & \left\{L_{f}\left(L_{f}+1\right)+S_{f}\left(S_{f}+1\right)-\frac{1}{4}\right. \\
& \left. \pm\left[\frac{4}{3} L_{f}\left(L_{f}+1\right) S_{f}\left(S_{f}+1\right)+\frac{1}{4}\right]^{1 / 2}\right\}-\frac{1}{2} .
\end{aligned}
$$

The LAD pattern, as well as the sum rules, are valid both for $\mathrm{LAD}(\cos )$ and $\mathrm{LAD}(\sin )$.

\section{Atoms with $P, D, F, \ldots$ symmetry}

In the general case $L_{0}>0$ the dichroism spectra may differ from the simple patterns shown in Fig. 2. In Sec. III A 2 we found that the dependence of the fine-structure integrated dichroism of atoms with $L_{0}>0$ on the angular momentum $L_{f}$ is formally equivalent to the dependence of dichroism of atoms with $L_{0}=0$ on the momentum $J_{f}$. Unfortunately, there is no such simple rule as the Lande interval rule for multiplets with different $L_{f}$ of one ionic configuration. For atoms with $P, D, F, \ldots$ symmetry the complete multiplet structure must be known in order to make any statement on the shape of dichroism in the photoelectron spectrum. In general, the simple spectral patterns shown in the panels on the top of Fig. 2 may be expected to show up for atoms with $P, D, F, \ldots$ symmetry only if the photoelectron lines with different angular momentum $L_{f}$ and fixed spin $S_{f}$ are arranged on the energy axis in a regular (ascending or descending) manner. An example will be considered in the following section. 


\section{EXPERIMENTAL EXAMPLES}

In this section we examine the results of recent experiments $[13,28,37,26]$ and compare them with the theoretical results presented above. In all of these experiments, free atoms in an atomic beam were prepared in an oriented or aligned ground state by optical pumping with circularly or linearly polarized laser radiation, respectively. The atoms polarized in this way were then ionized with linearly polarized synchrotron radiation, counterpropagating the laser radiation. The photoelectrons were analyzed with a $180^{\circ}$ cylindrical mirror analyzer with the spectrometer axis set parallel to the polarization vector of the undulator radiation. This setup in principle allows one to determine all the different types of dichroism displayed in Fig. 1.

\section{A. Nonresonant photoionization far above threshold}

Nonresonant $3 p$ photoelectron spectra of free $\mathrm{Cr}$ atoms [13] and $4 f$ photoelectron spectra of Eu atoms [28,29], displaying a marked magnetic dichroism, have been obtained recently; this work stimulated our interest in the inner-shell photoionization of polarized atoms far above the corresponding thresholds, in energy regions where no autoionization resonances are expected to occur. Both $\mathrm{Cr}$ and $\mathrm{Eu}$ are key elements because of their half-filled $3 d / 4 s$ and $4 f$ shells, respectively. According to Hund's rules, $\mathrm{Cr}$ and $\mathrm{Eu}$ have maximum spins of $S_{0}=3$ and $\frac{7}{2}$, respectively. In both cases the orbital momentum vanishes in the ground state $\left(L_{0}=0\right)$ and, therefore, $\mathrm{Cr}$ and Eu belong to the class of atoms with $S$ symmetry. The $3 p$ and $4 f$ spectra of $\mathrm{Cr}$ and Eu, respectively, will be considered in the following two sections before turning to the controversely discussed $3 p$ spectrum of Fe.

\section{The $3 p$ shell of $\mathrm{Cr}$}

Dichroism in the $3 p$ photoionization of $\mathrm{Cr}$ atoms has been extensively studied in recent years $[27,13,26]$. In the singleconfiguration approximation, the $3 p$ photoionization of $\mathrm{Cr}$ is described by

$$
\text { Cr } 3 p^{6} 3 d^{5} 4 s^{7} S_{3}+\gamma \rightarrow\left[3 p^{5} 3 d^{5} 4 s^{8,6} P+\varepsilon s, d\right]^{7} P \text {. }
$$

The ${ }^{8} P$ (binding energy $47 \mathrm{eV}$ ) and ${ }^{6} P$ (binding energy $>60$ $\mathrm{eV})$ components are separated by more than $10 \mathrm{eV}$ in the photoelectron spectrum [14]; the large exchange splitting is due to the strong coupling of the $3 p$ core hole to the halffilled $3 d$ shell. Here only the high-spin (low-binding energy) range will be considered.

The high-spin part of the photoelectron spectrum is displayed in the top part of Fig. 3. The spectrum was measured with the laser turned off and represents the photoionization cross section $\sigma$ [Eq. (7)]. It was taken at a photon energy of $\hbar \omega=76 \mathrm{eV}$, i.e. $30 \mathrm{eV}$ above the lowest ionization threshold. The three lines reflect the fine-structure splitting ${ }^{8} P_{5 / 2,7 / 2,9 / 2}$ of the $3 p$ hole state. Since the fine-structure splitting of less than $1 \mathrm{eV}$ is small compared to the exchange splitting of more than $10 \mathrm{eV}$, the $\mathrm{Cr} 3 p^{-1}$ spectrum provides a very good example for the $L S J$ coupling approximation. Indeed, the fine-structure splitting within the ${ }^{8} P$ line can be well described by the Lande interval rule; the relative intensities

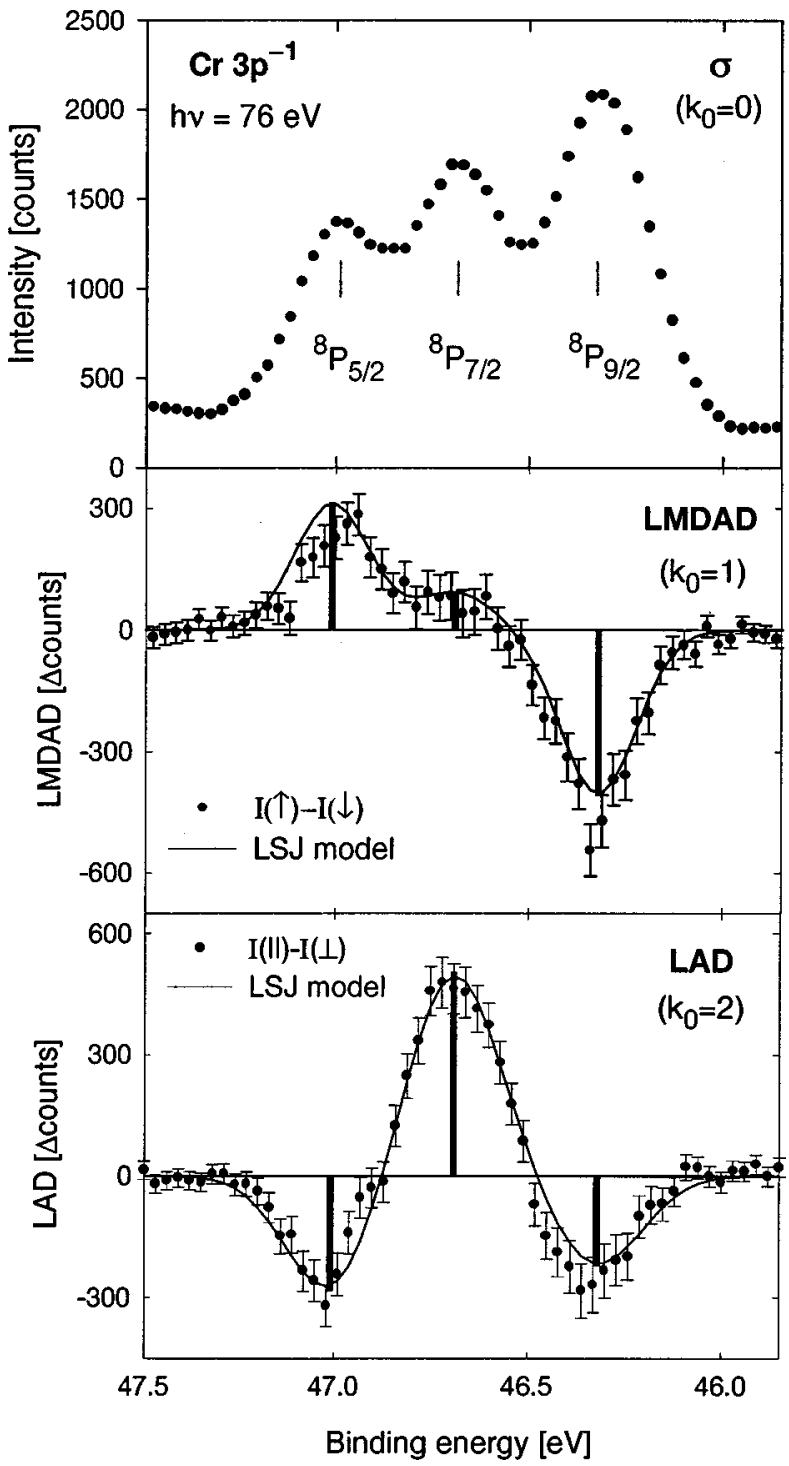

FIG. 3. Top part, photoelectron intensity in the $3 p^{6} 3 d^{5} 4 s^{7} S_{3}$ $+\gamma \rightarrow 3 p^{5} 3 d^{5} 4 s^{8} P_{5 / 2,7 / 2,9 / 2}+\varepsilon l$ main line, recorded at a photon energy of $76 \mathrm{eV}$ without laser; center part, difference of two spectra with mutually antiparallel atomic orientation (LMDAD); bottom part, difference of two spectra with mutually perpendicular atomic alignment (LAD). The experimental data were taken from Ref. [13]. The solid curves (-) in the center and bottom parts are based on the $L S J$ model and were generated from the fine-structure resolved bar spectrum as described in the text.

of the fine-structure components are very close to the statistical ratios of 3:4:5 expected in pure $L S J$ coupling.

The data points in the center panel of Fig. 3 give the linear magnetic dichroism LMDAD as defined in Fig. 1, i.e., the difference of two photoelectron spectra taken with mutually antiparallel orientations of the atoms. The data points in the lower panel of Fig. 3 give the linear alignment dichroism LAD, i.e., the difference of two spectra for parallel and perpendicular alignment of the atoms with respect to the polarization axis of the vuv radiation (it corresponds to $\mathrm{LAD}(\cos )$ as defined in Fig. 1). The solid curves in the center and bottom parts of Fig. 3 are based on the LSJ coupling model and were generated in the following way. Within the model introduced in Sec. II A the relative strength of LMDAD and 
LAD in the different fine-structure components $J_{f}$ is determined by the coupling coefficients $C_{1}^{S}$ and $C_{2}^{S}$, respectively, given by Eq. (15):

$$
\begin{array}{r}
\mathrm{LMDAD}, \quad I(\uparrow)-I(\downarrow) \propto C_{1}^{S}\left(3, J_{f}\right), \\
\mathrm{LAD}, \quad I(\|)-I(\perp) \propto C_{2}^{S}\left(3, J_{f}\right) .
\end{array}
$$

The length of the solid bars shown in both the LMDAD and the LAD spectrum is proportional to the coefficients $C_{1}^{S}$ and $C_{2}^{S}$, respectively; the position of the bars was matched to the experimental energies. The solid curve was obtained by assuming a Gaussian instrument function. The sum of the profiles was then matched to the experimental data by multiplication with a scaling factor.

From Fig. 3 it can be seen that there is good agreement between the measured dichroism and the model curve. In particular, the spectra clearly display the simple patterns of LMDAD and LAD predicted by theory (Sec. III B) with two/ three lobes of alternating signs and one/two zeros, respectively. We note that, in the present case, where the ionic fine structure is completely resolved, the dichroism patterns refer to the envelope of the different peaks in the dichroism spectrum. In the dichroism spectra of Fig. 3 there is no evidence for the contribution of higher state multipoles $\mathcal{A}_{30}, \mathcal{A}_{50}$ and $\mathcal{A}_{40}, \mathcal{A}_{60}$, respectively, which is in accordance with our model [see the discussion after Eq. (20)]. For both LMDAD and LAD the dichroism integrated over the three finestructure components vanishes within the experimental uncertainties in agreement with sum rule 1 (Sec. III A).

The LMDAD has also been measured in the second strongest line of the $\mathrm{Cr} 3 p^{-1}$ spectrum, which accompanies the dominating ${ }^{8} P$ line at $3 \mathrm{eV}$ higher binding energies [13]. The corresponding part of the photoelectron spectrum around $E_{b}=49 \mathrm{eV}$ is not covered by Fig. 3 of the present paper, but by Fig. 3 of Ref. [13]. This line is due to the final ionic state $\mathrm{Cr} 3 p^{5}\left(3 d^{5} D\right) 4 s^{6} P^{*}$, where the asterisk indicates that it is not the exchange partner of the ${ }^{8} P$ line since the $3 d^{5}$ shell recouples from ${ }^{6} S$ to ${ }^{4} D$ in the ionization process. At a photon energy of $\hbar \omega=103 \mathrm{eV}$ the fine structure was not resolved due to the finite bandpass of the analyzer. The LMDAD displays the same shape in the ${ }^{6} P^{*}$ line as in the ${ }^{8} P$ line, but with reversed sign along the energy axis. The appearance of the LMDAD pattern in the ${ }^{6} P^{*}$ line corroborates our finding that the qualitative description of dichroism with Eq. (15) is independent of the ionic parentage for atoms with $S$ symmetry (i.e., independent of the coupling of the $3 d$ shell in the case of $\mathrm{Cr}$ ), but only depends on the total quantum numbers $L_{f}, S_{f}$ of the final ionic state. The dichroism normalized to the partial cross section $\sigma$ should have approximately the same strength in the ${ }^{8} P$ and ${ }^{6} P^{*}$ lines according to Eqs. (14) $-(16)$, which is in reasonable agreement with experiment ([13], Fig. 3, bottom part). The opposite sign of the LMDAD in both lines yields the additional information that the ${ }^{6} P_{3 / 2,5 / 2,7 / 2}^{*}$ multiplet is inverted, which is in accordance with HF calculations $[13,14]$.

\section{The $4 f$ shell of $E u$}

The $4 f$ photoionization of Eu atoms in the singleconfiguration approximation reads

$$
\text { Eu } 4 f^{7} 6 s^{2}{ }^{8} S_{7 / 2}+\gamma \rightarrow\left[4 f^{6} 6 s^{27} F+\varepsilon d, g\right]^{8} P .
$$

In the final ionic state, the $4 f$-intrashell interaction amounts to $10 \mathrm{eV}$, while the spin-orbit interaction is about $160 \mathrm{meV}$ [29]. Therefore, the $L S J$ coupling approximation can be expected to hold well for the description of the Eu $4 f$ photoionization.

Comparing the $4 f$ photoionization of Eu with the $3 p$ photoionization of $\mathrm{Cr}$ is interesting because these two model systems, apart from their similarities (see the introduction of Sec. IV), differ systematically in many respects. First of all, the final ionic symmetries are different for $\mathrm{Cr}\left({ }^{8} \mathrm{P}\right)$ and Eu $\left({ }^{7} F\right)$. In particular, the $\mathrm{Cr}$ final ionic state consists of three fine-structure components, the Eu state of seven. Furthermore, in the case of Eu, the open $4 f$ shell itself was ionized, whereas in the $\mathrm{Cr}$ case the open $3 d$ shell acts as a spectator and the closed $3 p$ shell was ionized. Therefore, the photoelectron spectrum of the $\mathrm{Cr} 3 p$ shell is dominated by the $3 p-3 d$-intershell interaction, whereas for the $4 f$ ionization of $\mathrm{Eu}$, the $4 f$ intrashell interaction is dominating. Finally, the dynamics of photoionization is completely different for $\mathrm{Cr}$ and $\mathrm{Eu}$ at the respective photon energies. For $\mathrm{Eu}$ excited $38 \mathrm{eV}$ above threshold, the $g$ wave $\left(l_{>}=l_{0}+1\right)$ almost exclusively determines the $4 f$ photoionization cross section; the contribution of the $d$ wave $\left(l_{<}=l_{0}-1\right)$ is only $4 \%$ [29]. For Cr, on the other hand, the $s$ wave $\left(l_{<}\right)$dominates the $3 p$ photoionization cross section until far above threshold, since the $d$ wave $\left(l_{>}\right)$is suppressed by the centrifugal barrier and even goes through a Cooper minimum.

The Eu photoelectron spectrum in the binding energy region of the $4 f$ main line is depicted in the top part of Fig. 4. It was taken at a photon energy of $\hbar \omega=48 \mathrm{eV}$, i.e., well above the $4 f$ and also the $5 p$ thresholds. At these photon energies, $5 s$ electrons might be excited [38]; however, resonant enhancement of the photoelectron spectrum via $5 s$ excitations can be neglected since these resonances are weak and strongly broadened $[39,40]$. The instrumental bandwidth was $300 \mathrm{meV}$ and, therefore, the fine-structure splitting of the ${ }^{7} \mathrm{~F}$ multiplet of $\lesssim 140 \mathrm{meV}$ could not be resolved; only a broad asymmetric emission band is present. It should be noted that the lifetime broadening, in contrast to the instrumental broadening, is negligible since the final ionic states lie below the $\mathrm{Eu}^{2+}$ threshold at $E_{b}=17.9 \mathrm{eV}$ and thus cannot decay by Auger transitions.

The instrumental broadening, however, did not prevent the dichroism measurement in this line since the bandpass of the analyzer was still smaller than the total spread of the multiplet. The linear magnetic dichroism (LMDAD) [28] and the linear alignment dichroism (LAD) are shown in the center and bottom parts of Fig. 4. The theoretical spectra (solid lines) have been generated in the same way as for the $\mathrm{Cr}$ example. The height of the solid bars are proportional to the $L S J$ coupling coefficients $C_{k 0}^{S}$ [Eq. (15)]:

$$
\begin{array}{r}
\text { LMDAD, } \quad I(\uparrow)-I(\downarrow) \propto C_{1}^{S}\left(\frac{7}{2}, J_{f}\right), \\
\text { LAD, } \quad I(\|)-I(\perp) \propto C_{2}^{S}\left(\frac{7}{2}, J_{f}\right) .
\end{array}
$$

The relative energy positions of the bars were fixed according to Hartree-Fock calculations in intermediate coupling [29], a common energy shift was allowed. 


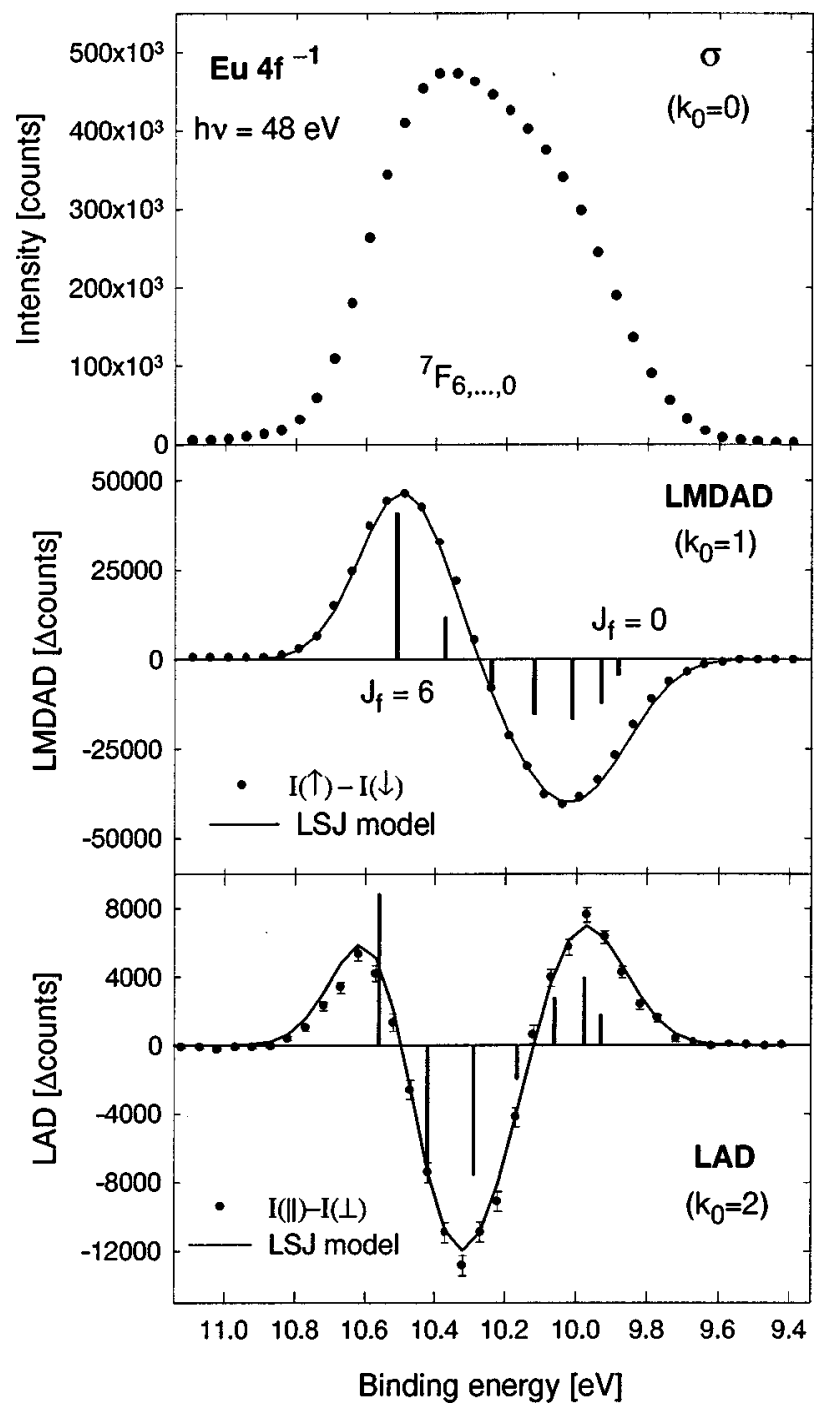

FIG. 4. Top part, photoelectron intensity in the $4 f^{7} 6 s^{2}{ }^{8} S_{7 / 2}$ $+\gamma \rightarrow 4 f^{6} 6 s^{2}{ }^{7} F+\varepsilon l$ main line, recorded at a photon energy of 48 $\mathrm{eV}$ without laser; center part, difference of two spectra with mutually antiparallel atomic orientation (LMDAD); bottom part, difference of two spectra with mutually perpendicular atomic alignment (LAD). The experimental data have partially been published in Ref. [28]. The solid curves (-) in the center and bottom parts are based on the $L S J$ model and were generated from the bar spectrum as described in the text.

The agreement between experiment and our theoretical model is excellent. The slight deviations for the LAD can partially be attributed to a larger statistical scatter compared to the LMDAD. The appearance of the dichroism patterns and especially the validity of the sum rule 1 can be proven very accurately for the $4 f$ photoionization of Eu. At this point, however, the contribution of higher state multipoles has to be discussed. For Eu, even in the nonrelativistic approximation state multipoles up to the rank $k_{0} \leqslant 6$ contribute to the dichroism. In the present case, although, for example, the magnitude of $\mathcal{A}_{40}$ is half that of $\mathcal{A}_{20}$, a careful investigation of the relevant factors has shown that the influence of higher state multipoles can be neglected [41].

In spite of the differences concerning the specific photoionization processes for $\mathrm{Cr}$ and $\mathrm{Eu}$ (see beginning of Sec. IV A 2), the dichroism patterns in the photoelectron spectra
(Figs. 3 and 4) are very similar. This similarity, which is independent in particular of the number of fine-structure components in the final ionic state, is well explained by the general results on the spectral form of dichroism obtained in Sec. III B. In addition to the $\mathrm{Eu} 4 f^{-1} \mathrm{LAD}(\cos )$ depicted in the lower part of Fig. 4, also the $\operatorname{LAD}(\sin )$ as defined in Fig. 1 has been measured. The shapes of both types of LAD are practically identical (while displaying the opposite sign) as is well explained by our model.

The measurements discussed here confirm that the $L S J$ approximation is well justified for both the $3 p$ emission of $\mathrm{Cr}$ and the $4 f$ emission of Eu. For the Eu $4 f^{-1}$ spectrum, however, deviations from pure $L S J$ coupling have been suggested ([32], p. 337) in order to explain deviations of the relative intensities of the multiplet components from their statistical weights. This problem is discussed in detail elsewhere [29]. The present dichroism measurements on Eu do not give any evidence for a direct influence of intermediate coupling on the dichroism, which should manifest itself as a significant deviation from sum rule 1 . On the other hand, we note that if (for the theoretical description) the fine-structure components of the Eu $4 f$ line are arranged on the energy axis according to the Landé interval rule (but still letting the total spread of the multiplet vary), the agreement between the experimental LMDAD and the fitted curve is poor, resulting in deviations up to $30 \%$.

It is stressed once more that all the theoretical curves of Figs. 3 and 4 have been generated without any knowledge of the magnitude of the respective dipole matrix elements. Within the experimental uncertainties we cannot find any evidence for the influence of the dynamics of photoionization, including relaxation, on the relative strength of dichroism in the photoelectron spectra. The overall sign of dichroism, however, is determined by the dynamical parameters $b_{k_{0} k k_{\gamma}}$ and cannot be predicted without the knowledge of the particular dipole matrix elements. Comparing, for example, Figs. 3 and 4, one notes that the LMDAD has the same sign but the LAD has indeed the opposite sign in each of the dichroism lobes. Similar considerations concern the strength of the dichroism normalized to the partial cross section $\sigma$. For $\mathrm{Eu}$, the normalized dichroism is $13 \%$ and for $\mathrm{Cr}$ it is $18 \%$ in the maximum of the LMDAD. A comparison of these numbers must take into account not only the different magnitudes of the atomic polarization and the different fraction of polarizable isotopes in the atomic beam ${ }^{3}[26,28]$, but also the different dynamical quantities $b_{k_{0} k_{\gamma}}$. In conclusion, the dynamics of photoionization within our model exclusively determines both the absolute scale and the overall sign of the dichroism in the photoelectron spectrum.

\section{The $3 p$ shell of $\mathrm{Fe}$}

Until now the dichroism in the vuv photoelectron spectrum of free $\mathrm{Fe}$ atoms has not been measured. In the present section it is our aim to make some qualitative predictions, based on an analysis of the photoelectron spectrum of unpolarized $\mathrm{Fe}$ atoms measured in the region of the dominating

\footnotetext{
${ }^{3}$ In the case of Eu, the measured LMDAD is furthermore lowered by $30 \%$ because of the limited instrumental resolution.
} 


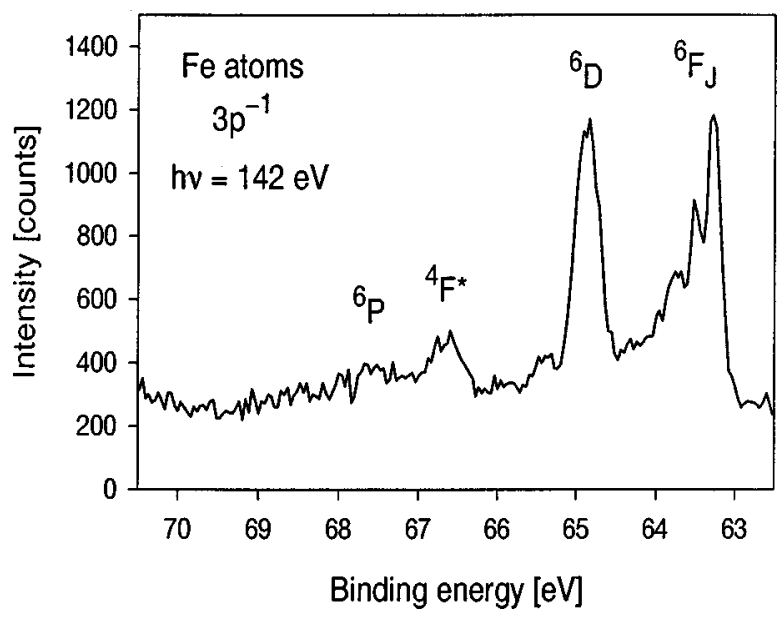

FIG. 5. Photoelectron spectrum of free Fe atoms in the region of the $3 p$ thresholds (taken from Ref. [37]).

$3 p^{-1}$ lines [37]; the spectrum is reproduced in Fig. 5 and has been taken at a photon energy of $\hbar \omega=142 \mathrm{eV}$ and an overall resolution of $\Delta E=0.2 \mathrm{eV}$.

Within the single-configuration approximation, the $3 p$ photoionization of atomic Fe can be written as

$$
\text { Fe } 3 p^{6} 3 d^{6} 4 s^{2}{ }^{5} D+\gamma \rightarrow 3 p^{5} 3 d^{6} 4 s^{24,6} P, D, F+\varepsilon l .
$$

As in the case of $\mathrm{Cr}$, the $3 p$ spectrum of $\mathrm{Fe}$ is characterized by the strong $3 p-3 d$-intershell coupling, which leads to an exchange splitting of $15 \mathrm{eV}$ between the ${ }^{6} X$ and ${ }^{4} X$ exchange partners $[43,14]$. Figure 5 shows the low binding energy part of the spectrum which covers the dominating high-spin ${ }^{6} X$ lines; the fine structure of the lowest line at $63.5 \mathrm{eV}$ assigned to ${ }^{6} F$ is partially resolved. The finestructure splitting is smaller than $0.3 \mathrm{eV}$ and, therefore, as for $\mathrm{Cr}$, the $L S J$ approximation is very well justified for Fe. The ${ }^{6} \mathrm{D}$ multiplet gives rise to the next line at $65.0 \mathrm{eV}$. The broad feature in the region $66-69 \mathrm{eV}$ is assigned to the ${ }^{6} P$ multiplet; the strong broadening is due to term-dependent Auger decay rates $[44,43,14]$.

The relative strength of the dichroism in the atomic Fe $3 p$ high-spin spectrum is given by the coupling coefficients $D_{k_{0}}\left(L_{f}\right)$, defined by Eq. (34), for the lines with different orbital angular momenta $L_{f}$ in the final ionic state. In the present context the most important finding from the experimental spectrum (Fig. 5) is that the three multiplets $L_{f}$ $=1,2,3$ of the high-spin spectrum $S_{f}=\frac{5}{2}$ are arranged in a regular manner on the energy axis, namely with descending binding energies $(67.5 \mathrm{eV}, 65 \mathrm{eV}, 63.5 \mathrm{eV})$. As a consequence of the mathematical dependence of the coefficients $D_{k_{0}}\left(L_{f}\right)$ on the angular momentum $L_{f}$ discussed in Sec. III B 2, the characteristic dichroism patterns both for CMD/ LMDAD (one zero) and LAD (two zeros) ${ }^{4}$ are to be expected in the low binding energy region of the photoelectron spectrum. In addition, the sum rule 2 is expected to hold.

\footnotetext{
${ }^{4}$ In the nonrelativistic approximation, higher state multipoles with $k_{0}>2$ do not contribute to the emission of a $p$ electron [see the discussion following Eq. (20)].
}

It should be noted that the assumption of a definite total angular momentum $J_{0}$ in the initial state is not well fulfilled in a hot atomic $\mathrm{Fe}$ beam. At $1580^{\circ} \mathrm{C}$, the temperature necessary for a vapor pressure of $10^{-2} \mathrm{~mm} \mathrm{Hg}$ [45], only half of the $\mathrm{Fe}$ atoms are in the state ${ }^{5} D_{4}$, the other half being thermally excited into the states ${ }^{5} D_{3-0}$. Although the dichroism patterns are obtained for any particular fine-structure component $J_{0}$ in the initial state (if considered separately), a rigid treatment would also have to involve possible interference effects for the case of hot $\mathrm{Fe}$ atoms. At room temperature (which is relevant for a comparison to the solid-state spectra discussed below) the assumption of a definite $J_{0}$ is valid since $90 \%$ of the Fe atoms are in the state ${ }^{5} D_{4}$.

In the low binding energy part of the atomic Fe $3 p$ spectrum, in addition to the high-spin lines ${ }^{6} X$, some low-spin lines ${ }^{4} X^{*}$ are also excited; the strongest multiplet ${ }^{4} F^{*}$ is marked in Fig. 5. These multiplets are analogous to the recoupled ${ }^{6} P^{*}$ line in the $\mathrm{Cr} 3 p$ spectrum which was discussed above. The ${ }^{4} F^{*}$ line in Fig. 5 is due to a recoupling of the Fe $3 d^{6}$ shell from ${ }^{5} D$ to ${ }^{3} F$ in the photoionization process. These lines are not the exchange partners of the ${ }^{6} X$ lines; the ${ }^{4} X$ lines are expected at much higher binding energies. The calculated oscillator strength of the ${ }^{4} X^{*}$ multiplets relative to that of the ${ }^{6} X$ multiplets is $15 \%[43,14]$; even the strongest of the ${ }^{4} X^{*}$ multiplets $\left({ }^{4} F^{*}\right)$ is only half as strong as the weakest of the ${ }^{6} X$ multiplets $\left({ }^{6} P\right)$. Therefore, we expect that the dichroism in the low-binding energy region of the atomic Fe $3 p$ spectrum is dominated by the contribution of the ${ }^{6} X$ multiplets; the disturbance, due to the contribution of the ${ }^{4} X^{*}$ multiplets, should be weak. In particular, the gross appearance of the characteristic dichroism patterns with two or three lobes in the low binding energy region of the atomic spectrum should be preserved, and the dichroism integrated over that energy region should vanish.

In the second part of this section we address the dichroism in the $3 p$ spectrum of $\mathrm{Fe}$ atoms bound to a surface. The $3 p$ spectrum of Fe metal [46] displays a strong main line at a binding energy of $52.5 \mathrm{eV}$ with a width of $3 \mathrm{eV}$, which is somewhat narrower than the total spread of $5 \mathrm{eV}$ of the atomic high-spin multiplet. Taking into account the very strong exchange interaction, this main line should belong mostly to the high-spin final ionic states $[47,48]$, with a possible contribution from the recoupled low-spin satellite multiplet discussed above. It is remarkable that both the LMDAD and the LD in this main line, measured in the pioneering experiment of Roth et al. [[46] Figs. 3(c) and 2(b)], clearly display the characteristic dichroism patterns that we also predict for the atomic case. Nevertheless, we are aware that an interpretation of the $\mathrm{Fe} 3 p$ surface spectrum within a purely atomic model falls short of important solidstate aspects, for example, the partially itinerant character of the $3 d$ electrons in the condensed phase and the symmetry breaking due to the crystal field. In the following, some effects of the solid-state environment for the dichroism are discussed.

The occupation number of the $3 d$ shell of Fe in the bulk ground state is not equal to the atomic value 6 , but intermediate between 6 and 7 [49]. Furthermore, in the final ionic state the $3 d$ occupation number is affected by screening and relaxation effects in the innershell ionization process. At least for integer initial configurations $3 d^{7}$ (atomic Co) and 
$3 d^{8}$ (atomic Ni), our model (making use of the experimental $\mathrm{Co}$ and $\mathrm{Ni} 3 p^{-1}$ multiplet structure $[14,50]$ ) predicts the persistance of the characteristic dichroism patterns with two or three lobes, respectively, in the high-spin part of the spectrum. Therefore, it seems that neither the appearance of the simple dichroism patterns nor the validity of the sum rule are sensitive to the number of $3 d$ electrons.

Furthermore, the magnetic moment per atom of $\mathrm{Fe}$ metal in the ground state is $2.3 \mu_{B}$, which is significantly less than one would expect from a ${ }^{5} \mathrm{D}$ ground state of the ionic $\mathrm{Fe}$ cores, even if the contribution of the orbital angular momentum is neglected because of orbital quenching. Here we note that within our model, neither the appearance of the dichroism patterns nor the validity of the sum rule depend on the values of the initial and final ionic spins $S_{0}$ and $S_{f}$.

From the above we conclude that a qualitative understanding of the dichroism in the solid-state Fe $3 p$ photoelectron spectra is possible within the atomic $L S J$ coupling model. Our approach is very similar to that of Bagus and Mallow [48] and Kachel et al. [47] who described the spinpolarized solid $\mathrm{Fe} 3 p$ photoelectron spectra. On the other hand, a description within a one-electron picture meets certain difficulties. In a one-electron picture, the $3 p-3 d$ exchange interaction is modeled by a spin-dependent field ${ }^{5} H_{s}$. Van der Laan [52] calculated the LMDAD and LD in the Fe $3 p$ spectrum in a one-electron model; intermediate coupling was assumed and the exchange interaction was modeled by a spin field of $H_{s}=1.3 \mathrm{eV}$, being approximately as large as the spin-orbit splitting of the $3 p$ core hole of $\zeta_{s}(3 p)=0.95 \mathrm{eV}$. The result ([52], Fig. 9) deviates from experiment; it displays two zeros instead of one for the LMDAD, and four zero instead of two for the LD.

A single-electron model was also used by Cherepkov et al. $[53,10]$ in order to explain the dichroism in the Fe $3 p$ spectrum. In Ref. [10] the LAD in the solid $\mathrm{Fe} 3 p$ main line was approximated by four Lorentzian profiles corresponding to the fine-structure components of the $3 p_{3 / 2}$ core hole. In Ref. [53] the LMDAD in the solid Fe $3 p$ main was approximated line by six profiles corresponding to the fine-structure components of the $3 p_{3 / 2,1 / 2}$ core hole. The contribution of the $3 p_{1 / 2}$ component, although masked by the contribution of the $3 p_{3 / 2}$ component, still leads to a second zero in the theoretical LMDAD spectrum at a binding energy of $54.7 \mathrm{eV}$ ([53], Fig. 3), which is not reproduced by experiment [46,53]. As was noted above, within LSJ coupling, the low-spin exchange partner lies at much higher binding energies leaving only one zero for the LMDAD in the strong main line due to the high-spin component.

\section{B. Resonantly enhanced photoionization near threshold}

It is instructive to contrast the results obtained above with the case of near threshold excitation, i.e., in the region of autoionization resonances [26,54]. For photon energies near threshold, resonances with well-defined total momentum $J$ can be excited, effectively filtering out one of the possible $J$

\footnotetext{
${ }^{5}$ In contrast, the inner magnetic field induced by the valence electrons at the $\mathrm{Fe}$ cores gives rise to a splitting of the order of $1 \mathrm{meV}$ according to Mössbauer spectra ([51], p. 163).
}

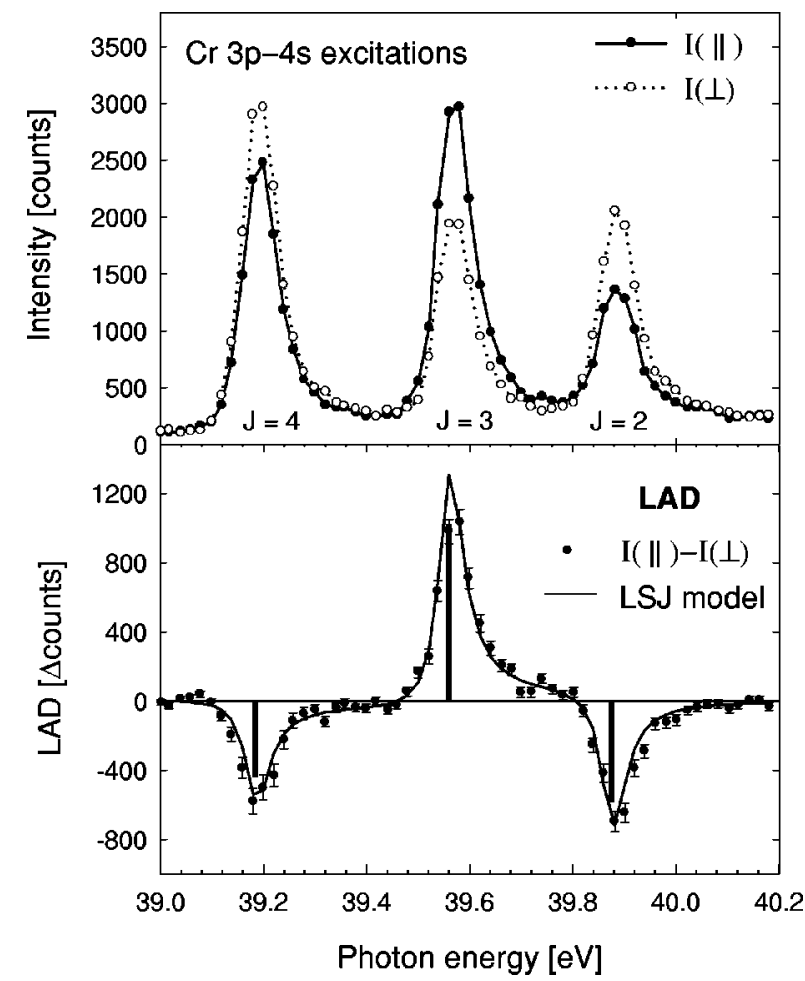

FIG. 6. Upper part, partial $\mathrm{Cr} 3 d^{-1}$ photoionization cross section in the region of the $3 p \rightarrow 4 s$ inner-shell resonances for parallel $(\bullet)$ and perpendicular $(\circ)$ alignment of the atoms relative to the polarization vector of the vuv radiation. Lower part, linear alignment dichroism (LAD) given by the difference of the two curves shown above. The experimental data were taken from Ref. [26]. The solid curve (-) is based on the LSJ model and was generated from the bar spectrum as described in the text.

values in the final (ion+electron) state. Therefore, the summation over $J J^{\prime}$ in Eq. (5) is no longer valid, but the resonance approximation $J=J^{\prime}$ has to be considered.

A good example is provided by the $3 p \rightarrow 4 s$ inner-shell excitations of aligned $\mathrm{Cr}$ atoms in the photon energy region of $39-40 \mathrm{eV}$. The measured $3 d^{-1}$ partial photoionization cross section [26], shown in the upper part of Fig. 6, displays three separated resonances which are assigned to the $3 p$ hole states $\operatorname{Cr} 3 p^{5}\left(3 d^{56} S_{5 / 2}\right) 4 s^{27} P_{J}$ with $J=2,3,4$. The two curves in the upper part of Fig. 6 correspond to parallel/ perpendicular alignment of the atoms with respect to the polarization vector of the vuv radiation. A strong dichroism is indeed observed, although the fine structure in the final ionic state $\operatorname{Cr} 3 p^{6} 3 d^{4} 4 s^{6} D_{1 / 2, \ldots, 9 / 2}$ was not resolved. More generally, it can be concluded that in order to observe a dichroism in the case of $L S J$ coupling, it is necessary to discriminate between either the open or the closed fine-structure channels.

The difference spectrum in the lower part of Fig. 6 gives the LAD. The most important result is that the spectral pattern of LAD also shows up in the partial cross section, i.e., on the photon energy axis (while up to this point only electron energy spectra have been considered). In particular, the LAD integrated over the $3 p \rightarrow 4 s$ resonances is accurately zero. In order to theoretically describe the dichroism in resonant cross sections, we substitute Eq. (10) into Eq. (5), perform the summations over $j, j^{\prime}, J_{f}$, insert $J=J^{\prime}, L_{0}=0$, and finally obtain 


$$
\begin{aligned}
B_{k_{0} k k_{\gamma}}= & 3 \hat{S}_{0}^{-1} \hat{J}^{4}(-1)^{k_{\gamma}+J+L_{f}+S_{0}} \\
& \times\left\{\frac{1}{2} S_{f} S_{0}\right\}\left\{\begin{array}{ccc}
k & 1 & 1 \\
S_{0} & J & J
\end{array}\right\}\left\{\begin{array}{ccc}
S_{0} & 1 & J \\
S_{0} & 1 & J \\
k_{0} & k_{\gamma} & k
\end{array}\right\} \\
& \times \sum_{l l^{\prime}} \hat{l} \hat{l}^{\prime}\left(l 0, l^{\prime} 0 \mid k 0\right)\left\{\begin{array}{ccc}
k & 1 & 1 \\
L_{f} & l & l^{\prime}
\end{array}\right\} D_{l 1 S_{0}} D_{l^{\prime} 1 S_{0}}^{*} .
\end{aligned}
$$

From a triangular rule for the $6 j$ symbols in Eq. (46), it generally follows that only multipoles up to $k \leqslant 2$ in the photoelectron angular distribution contribute. Analogous to Eq. (19), the dependence of the $B_{k_{0} k k_{\gamma}}$ parameters [Eq. (46)] on the total angular momentum $J$ is completely separated from the dipole amplitudes. However, in contrast to Eq. (19), in the present case the angular-momentum coupling coefficients depend, in addition to the rank $k_{0}$ of the statistical tensors, also on $k_{\gamma}$ and $k$. Therefore, the dichroism in the resonance photoionization cross section in general will be different from the simple patterns obtained in Sec. III B.

As an important consequence of the resonance approximation, there is no sum rule analogous to sum rule 1 given in Sec. III A in the present case; the sum of the $B_{k_{0} k k_{\gamma}}$ parameters [Eq. (46)], over the fine structure $J$ of the autoionizing multiplet does not vanish because the interference terms with $J^{\prime} \neq J$ are missing. Only if the interference terms do not contribute for other reasons, such as for example, in an angleintegrating geometry (absorption or electron/ion yield experiments), an analogous sum rule is valid. The interference in $J$ vanishes if only terms with $k=0$ contribute to the dichroism, as can be seen from a triangle rule for the $9 j$ symbol in Eq. (5); for $k=0$, Eq. (46) is considerably simplified:

$$
\begin{aligned}
B_{k_{0} 0 k_{\gamma}}= & \delta_{k_{0} k_{\gamma}} \hat{k}_{0}^{-1} \hat{J}^{2} \hat{S}_{0}^{-1}(-1)^{S_{0}+J+1} \\
& \times\left\{\frac{1}{2} S_{f} S_{0}\right\}\left\{\begin{array}{ccc}
S_{0} & S_{0} & k_{0} \\
1 & 1 & J
\end{array}\right\} \sum_{l}\left|D_{l 1 S_{0}}\right|^{2} .
\end{aligned}
$$

The dependence of Eq. (47) on the angular momentum $J$ is equivalent to that of Eq. (23) on $J_{f}$ which establishes ${ }^{6}$ the appearance of the characteristic dichroism patterns (two/ three lobes), as well as the validity of the sum rule, in the resonant photoionization cross section. Indeed, the solid line

\footnotetext{
${ }^{6}$ In addition to the nonresonant case where the photon energy is fixed, here we have to assume that the dependence on the singleelectron dipole amplitudes on the photon energy is negligible over the small region of the photoionization cross section of an autoionizing multiplet.
}

in Fig. 6, which has been generated from the solid bar spectrum [determined in turn by Eq. (47)] in the same way as before, describes the LAD pattern very well. This is because, for the experimental geometry used for the LAD measurements displayed in Fig. 6, the condition $k \leqslant 2$ in fact implies $k=0$ [[41], Eq. (2.33) and [18], Eqs. (15) and (16)]. In contrast to the dichroism in the photoelectron spectrum, Eq. (47) predicts even the absolute sign of the dichroism in the different fine-structure components of an autoionizing multiplet. Finally, it should be noted that the appearance of the magnetic dichroism pattern (two lobes) in an angleintegrating CMD measurement is to be expected as well.

\section{CONCLUSION}

We have shown that, in the nonrelativistic approximation and for photon energies far above threshold, the generalized anisotropy coefficients, which determine the angular distribution of photoelectrons emitted from polarized atoms can be presented in a product form; one of the factors completely contains the dependence on the total angular momenta of the target atom and the final ion. As a consequence, the spectral distribution of dichroism turns out to have general properties as a function of the photoelectron energy, charateristic to each type of dichroism (CMD, LMDAD, and LAD). Simple dichroism patterns are obtained which are practically independent of the photoionization dynamics and of the finalstate multiplicity.

Two sum rules are derived which characterize the dichroism integrated over the energy interval of one or several final ionic multiplets. The sum rules may be convenient for testing the consistency of theoretical calculations or the validity of the approximations made.

For illustration we have analyzed the LMDAD and the LAD in the $3 p$ and $4 f$ photoionization of atomic $\mathrm{Cr}$ and $\mathrm{Eu}$, respectively. The experimental data are very well described by the characteristic patterns obtained here. A qualitative analysis was performed for $3 p$ photoionization of $\mathrm{Fe}$ atoms on a solid surface; we have presented arguments that the observed dichroism patterns are strongly influenced by intraatomic effects.

\section{ACKNOWLEDGMENTS}

We thank B. Sonntag and P. Zimmermann for stimulating discussions and helpful advice. We are thankful to J. E. Hansen and M. Martins for the atomic structure calculations on $\mathrm{Fe}$ and $\mathrm{Eu}$, respectively, and to K. J. Ross for a careful reading of the manuscript. A.V. acknowledges financial support by the Deutsche Forschungsgemeinschaft (DFG So 176/ 2). A.N.G. gratefully acknowledges the hospitality of the University Hamburg and the Albert-Ludwigs-University Freiburg. N.M.K. is grateful for hospitality extended to him during his stay at the University Bielefeld where part of this work was done.
[1] H. Klar and H. Kleinpoppen, J. Phys. B 15, 933 (1982).

[2] N. A. Cherepkov, V. V. Kuznetsov, and V. A. Verbitskii, J. Phys. B 28, 1221 (1995).

[3] C. Kerling, N. Böwering, and U. Heinzmann, J. Phys. B 23, L629 (1990).
[4] O. Plotzke, G. Prümper, B. Zimmermann, U. Becker, and H. Kleinpoppen, Phys. Rev. Lett. 77, 2642 (1996).

[5] G. Prümper, B. Zimmermann, B. Langer, N. A. Cherepkov, U. Becker, and H. Kleinpoppen (unpublished).

[6] K. Godehusen, P. Zimmermann, A. Verweyen, A. von dem 
Borne, P. Wernet, and B. Sonntag, Phys. Rev. A 58, R3371 (1998).

[7] J. G. Menchero, Phys. Rev. B 57, 993 (1998).

[8] G. van der Laan, E. Arenholz, E. Navas, A. Bauer, and G. Kaindl, Phys. Rev. B 53, R5998 (1996).

[9] F. U. Hillebrecht, Ch. Roth, H. B. Rose, W. G. Park, E. Kisker, and N. A. Cherepkov, Phys. Rev. B 53, 12182 (1996).

[10] N. A. Cherepkov, Phys. Rev. B 50, 13813 (1994).

[11] B. T. Thole and G. van der Laan, Phys. Rev. B 49, 9613 (1994).

[12] H. Ebert, L. Baumgarten, C. M. Schneider, and J. Kirschner, Phys. Rev. B 44, 4406 (1991).

[13] A. von dem Borne, T. Dohrmann, A. Verweyen, B. Sonntag, K. Godehusen, and P. Zimmermann, Phys. Rev. Lett. 78, 4019 (1997).

[14] A. von dem Borne, R. L. Johnson, J. Schulz, B. Sonntag, M. Talkenberg, A. Verweyen, Ph. Wernet, K. Tiedtke, Ch. Gerth, B. Obst, P. Zimmermann, and J. E. Hansen, J. Phys. B (to be published).

[15] S. Baier, A. N. Grum-Grzhimailo, and N. M. Kabachnik, J. Phys. B 27, 3363 (1994).

[16] G. Schöhense and J. Hormes, in VUV and Soft X-Ray Photoionization, edited by U. Becker and D. A. Shirley (Plenum, New York, 1996), p. 607.

[17] L. Baumgarten, C. M. Schneider, H. Petersen, F. Schäfers, and J. Kirschner, Phys. Rev. Lett. 65, 492 (1990).

[18] M. Wedowski, K. Godehusen, F. Weisbarth, P. Zimmermann, M. Martins, Th. Dohrmann, A. von dem Borne, B. Sonntag, and A. N. Grum-Grzhimailo, Phys. Rev. A 55, 1922 (1997).

[19] N. Manakov, S. Marmo, and A. Meremianin, J. Phys. B 29, 2711 (1996).

[20] S. Schohl, D. Klar, N. A. Cherepkov, I. D. Petrov, K. Ueda, S. Baier, R. Kau, and H. Hotop, J. Phys. B 30, 609 (1997).

[21] S. Schohl, N. A. Cherepkov, I. D. Petrov, V. L. Sukhorukov, S. Baier, and H. Hotop, J. Phys. B 31, 3363 (1998).

[22] D. A. Varshalovich, A. N. Moskalev, and V. K. Khersonskii, Quantum Theory of Angular Momentum (World Scientific, Singapore, 1988).

[23] N. A. Cherepkov and V. V. Kuznetsov, J. Phys. B 22, L405 (1989).

[24] C. Pan and A. F. Starace, Phys. Rev. A 47, 295 (1993).

[25] S. T. Manson and A. F. Starace, Rev. Mod. Phys. 54, 389 (1982).

[26] Th. Dohrmann, A. von dem Borne, A. Verweyen, B. Sonntag, M. Wedowski, K. Godehusen, and P. Zimmermann, J. Phys. B 29, 5699 (1996).

[27] A. von dem Borne, Th. Dohrmann, A. Verweyen, B. Sonntag, K. Godehusen, P. Zimmermann, and N. M. Kabachnik, J. Phys. B 31, L41 (1998).

[28] A. Verweyen, Ph. Wernet, B. Sonntag, K. Godehusen, and P. Zimmermann, J. Electron Spectrosc. Relat. Phenom. 101-103, 179 (1999).
[29] A. Verweyen, Ph. Wernet, J. Schulz, B. Sonntag, M. Martins, K. Godehusen, and P. Zimmermann, J. Phys. B (to be published).

[30] I. I. Sobel'man, Introduction to the Theory of Atomic Spectra (Pergamon, Oxford, 1972).

[31] A. F. Starace, in Handbuch der Physik, Bd. 31, edited by W. Mehlhorn (Springer-Verlag, Berlin, 1982), p. 1.

[32] H. Siegbahn and L. Karlsson, in Handbuch der Physik, Bd. 31 (Ref. [31]), p. 215.

[33] B. T. Thole, P. Carra, F. Sette, and G. van der Laan, Phys. Rev. Lett. 68, 1943 (1992).

[34] P. Carra, B. T. Thole, M. Altarelli, and X. Wang, Phys. Rev. Lett. 70, 694 (1993).

[35] B. T. Thole and G. van der Laan, Phys. Rev. Lett. 70, 2499 (1993).

[36] G. van der Laan and B. T. Thole, Phys. Rev. B 48, 210 (1993).

[37] Ch. Gerth, K. Tiedtke, M. Martins, B. Obst, P. Zimmermann, P. Glatzel, A. Verweyen, Ph. Wernet, and B. Sonntag, J. Phys. B 31, 2539 (1998).

[38] M. Richter, M. Meyer, M. Pahler, T. Prescher, E. v. Raven, B. Sonntag, and H.-E. Wetzel, Phys. Rev. A 40, 7007 (1989).

[39] E. J. McGuire, Phys. Rev. A 10, 13 (1974).

[40] R. L. Cohen, G. K. Wertheim, A. Rosencwaig, and H. J. Guggenheim, Phys. Rev. B 5, 1037 (1972).

[41] A. Verweyen, Ph.D. thesis, Universität Hamburg, 1998.

[42] S. Gorovikov, S. Bode, K. Starke, G. Kaindl, and G. van der Laan, BESSY annual report, 1997, p. 303.

[43] J. E. Hansen (unpublished).

[44] E. J. McGuire, Phys. Rev. A 10, 32 (1974).

[45] K. J. Ross and B. Sonntag, Rev. Sci. Instrum. 66, 4409 (1995).

[46] Ch. Roth, H. B. Rose, F. U. Hillebrecht, and E. Kisker, Solid State Commun. 86, 647 (1993).

[47] T. Kachel, C. Carbone, and W. Gudat, Phys. Rev. B 47, 15391 (1993).

[48] P. S. Bagus and J. V. Mallow, Chem. Phys. Lett. 228, 695 (1994).

[49] V. L. Moruzzi, J. F. Janak, and A. R. Williams, Calculated Electronic Properties of Metals (Pergamon, New York, 1978).

[50] K. Tiedtke, Ch. Gerth, B. Kanngießer, B. Obst, P. Zimmermann, M. Martins and A. Tutay, Phys. Rev. A (to be published).

[51] H. Wegener, Der Mössbauer-Effekt und seine Anwendung in Physik und Chemie (Bibliographisches Institut, Mannheim, 1965).

[52] G. van der Laan, Phys. Rev. B 51, 240 (1995).

[53] G. Rossi, F. Sirotti, N. A. Cherepkov, F. Combet Farnoux, and G. Panaccione, Solid State Commun. 90, 557 (1994).

[54] G. Prümper, B. Zimmermann, B. Langer, O. Plotzke, M. Martins, K. Wielizcek, A. Hempelmann, M. Wiedenhöft, U. Becker, and H. Kleinpoppen, J. Phys. B 30, L683 (1997). 